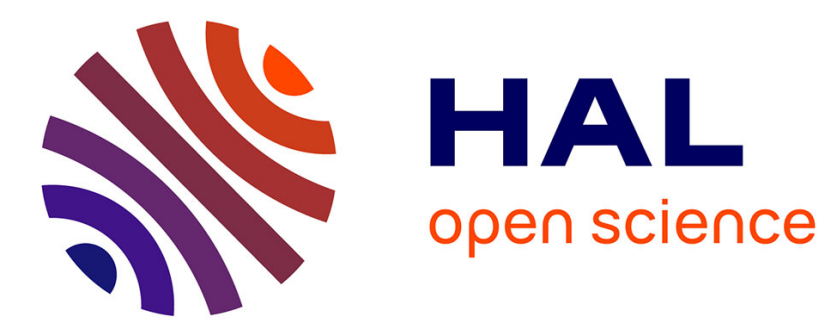

\title{
Error analysis of a subgrid eddy viscosity multi-scale discretization of the Navier-Stokes equations
}

\author{
Christine Bernardi, Tomas Chacon-Rebollo, Macarena Gómez Marmol
}

\section{To cite this version:}

Christine Bernardi, Tomas Chacon-Rebollo, Macarena Gómez Marmol. Error analysis of a subgrid eddy viscosity multi-scale discretization of the Navier-Stokes equations. 2012. hal-00684976

\section{HAL Id: hal-00684976 \\ https://hal.science/hal-00684976}

Submitted on 3 Apr 2012

HAL is a multi-disciplinary open access archive for the deposit and dissemination of scientific research documents, whether they are published or not. The documents may come from teaching and research institutions in France or abroad, or from public or private research centers.
L'archive ouverte pluridisciplinaire HAL, est destinée au dépôt et à la diffusion de documents scientifiques de niveau recherche, publiés ou non, émanant des établissements d'enseignement et de recherche français ou étrangers, des laboratoires publics ou privés. 


\title{
Error analysis
}

\section{of a subgrid eddy viscosity multi-scale discretization of the Navier-Stokes equations}

\author{
by Christine Bernardi ${ }^{1}$, Tomás Chacón Rebollo ${ }^{2}$, and Macarena Gómez Mármol²
}

\begin{abstract}
We propose a finite element discretization of the Navier-Stokes equations that relies on the variational multi-scale approach together with the addition of a Smagorinsky type viscosity, in order to take into account possible subgrid turbulence. We recall that the discrete problem admits a solution and prove a priori error estimates. Next we perform the a posteriori analysis of the discretization. Some numerical experiments justify the interest of this approach.
\end{abstract}

Résumé: Nous proposons une discrétisation par éléments finis des équations de NavierStokes qui fait appel à la méthode variationnelle multi-échelles et incorpore une viscosité de type Smagorinsky, de façon à prendre en compte d'éventuels phénomènes de turbulence. Nous rappelons l'existence d'une solution pour le problème discret et nous établissons des estimations d'erreur a priori. Puis nous effectuons l'analyse a posteriori de la discrétisation. Quelques expériences numériques confirment l'intérêt de cette approche.

1 Laboratoire Jacques-Louis Lions, C.N.R.S. \& Université Pierre et Marie Curie - Paris 6, boîte 187, 4 place Jussieu, 75252 Paris Cedex 05, France.

bernardi@ann.jussieu.fr

2 Departamento de Ecuaciones Diferenciales y Análisis Numerico, Universidad de Sevilla, Tarfia s/n, 41012 Sevilla, Spain.

chacon@us.es, macarena@us.es 



\section{Introduction.}

This paper deals with the numerical approximation of incompressible flows in turbulent regime by means of grid adaptation techniques.

Grid adaptation techniques are currently used to solve fluid flow problems, providing large savings of computational complexity. The numerical analysis of this technique is largely based upon a well-sound mathematical analysis of the problem considered. However, the mathematical analysis of many standard turbulence models is not well developed up-to-date. Even more, some commonly used models, in particular the $\mathrm{k}-\varepsilon$ one, do not seem to be well posed from the mathematical point of view.

An emerging class of turbulence models that is increasingly used due to its accuracy and simplicity is provided by the Variational Multi-Scale (VMS) setting (see [16] for a general description of VMS models). This is a fully discrete model, that does not require a continuous modeling step. The VMS procedure yields a discrete equation for a finite element approximation of the flow, where the eddy viscosity only acts on the small resolved scales of the flow. A simple modeling of the eddy viscosity acting on these scales (the Smagorinsky model) is used. This kind of combination of variational multi-scale and Smagorinsky models for small scales yields numerical results similar to those provided by up-to-date Large Eddy Simulation (LES) models (see [17], [18], [19]).

We consider in this paper a VMS-Smagorinsky turbulence model for which we perform a numerical analysis that extends the usual one for standard discretizations of NavierStokes equations.

Let $\Omega$ be a bounded connected domain in $R^{d}, d=2$ or $d=3$, with a Lipschitzcontinuous boundary $\partial \Omega$. We are interested in the finite element discretization of the Navier-Stokes equations in this domain relying on the variational multi-scale method, in cases where turbulence phenomena can occur. More precisely,

(i) For both the velocity and the pressure, we introduce two spaces of discretization, one called coarse and the other one called fine: Indeed, the fine space either is defined from a mesh which is refined from the mesh used for the coarse space or involves higher degree polynomials.

(ii) A further nonlinear viscosity term is added. In standard tubulence models, this viscosity can depend on other unknowns which are the solution of convection-diffusion equations, for instance the turbulent kinetic energy (see [2] for instance), or the temperature (see [3] for instance). Here, we have chosen to work with the simpler and well-known nonlinear Smagorinsky viscosity, introduced in [22].

We refer to [8, Chap. 2] for a complete description of this discretization which brings to light its interest for the approximation of turbulent flows.

We thus consider a variational multi-scale approximation of Navier-Stokes equations in turbulent regime for which the Smagorinsky eddy viscosity acts only on the small resolved scales of the flow. In a rather general finite element framework, relying on standard 
arguments for nonlinear problems, we perform the a priori and a posteriori analysis of the discrete problem. This leads to optimal error estimates. A few numerical experiments are in good coherence with the theoretical results.

An outline of the paper is as follows.

- In Section 2, we present the continuous and discrete problems we work with and recall their main properties.

- Section 3 and 4 are devoted to the a priori and a posteriori analysis of the discretization, respectively.

- Numerical experiments are presented in Section 5.

- The final section presents the main conclusions of the paper. 


\section{The continuous and discrete problems.}

We have decided for simplicity to work with homogeneous no-slip boundary conditions. In this case, the Navier-Stokes equations in $\Omega$ read

$$
\begin{cases}-\nu \Delta \boldsymbol{u}+(\boldsymbol{u} \cdot \nabla) \boldsymbol{u}+\operatorname{grad} p+\boldsymbol{f} & \text { in } \Omega, \\ \operatorname{div} \boldsymbol{u}=0 & \text { in } \Omega, \\ \boldsymbol{u}=\mathbf{0} & \text { on } \partial \Omega .\end{cases}
$$

The unknowns are the velocity $\boldsymbol{u}$ and the pressure $p$ of the fluid. The data are only the distribution $\boldsymbol{f}$ which represents a density of body forces, while the viscosity $\nu$ of the fluid is a positive constant.

\subsection{Variational formulation of the continuous problem.}

We consider the full scale of Sobolev spaces $H^{s}(\Omega), s \in \mathbb{R}$, and $W^{m, p}(\Omega), m \in \mathbb{N}$, $1 \leq p \leq \infty$, equipped with the standard norms and seminorms. In order to write a variational formulation of problem (2.1), we also introduce the space $H_{0}^{1}(\Omega)$ of functions in $H^{1}(\Omega)$ vanishing on $\partial \Omega$ and its dual space $H^{-1}(\Omega)$. We finally need the space

$$
L_{\circ}^{2}(\Omega)=\left\{q \in L^{2}(\Omega) ; \int_{\Omega} q(\boldsymbol{x}) \mathrm{d} \boldsymbol{x}=0\right\} .
$$

Standard density results yield that system (2.1) (where the first two lines are satisfied in the distribution sense) is fully equivalent to the following variational problem

Find $(\boldsymbol{u}, p)$ in $H_{0}^{1}(\Omega)^{d} \times L_{\circ}^{2}(\Omega)$ such that

$$
\begin{aligned}
& \forall \boldsymbol{v} \in H_{0}^{1}(\Omega)^{d}, \quad a(\boldsymbol{u}, \boldsymbol{v})+c(\boldsymbol{u}, \boldsymbol{u}, \boldsymbol{v})+b(\boldsymbol{v}, p)=\langle\boldsymbol{f}, \boldsymbol{v}\rangle, \\
& \forall q \in L_{\circ}^{2}(\Omega), \quad b(\boldsymbol{u}, q)=0,
\end{aligned}
$$

where $\langle\cdot, \cdot\rangle$ stands for the duality pairing between $H^{-1}(\Omega)^{d}$ and $H_{0}^{1}(\Omega)^{d}$. The bilinear forms $a(\cdot, \cdot)$ and $b(\cdot, \cdot)$ are defined by

$$
a(\boldsymbol{u}, \boldsymbol{v})=\nu \int_{\Omega}(\operatorname{grad} \boldsymbol{u})(\boldsymbol{x}):(\operatorname{grad} \boldsymbol{v})(\boldsymbol{x}) \mathrm{d} \boldsymbol{x}, \quad b(\boldsymbol{v}, q)=-\int_{\Omega}(\operatorname{div} \boldsymbol{v})(\boldsymbol{x}) q(\boldsymbol{x}) \mathrm{d} \boldsymbol{x}
$$

while the trilinear form $c(\cdot, \cdot, \cdot)$ is given by

$$
c(\boldsymbol{w}, \boldsymbol{u}, \boldsymbol{v})=\int_{\Omega}((\boldsymbol{w} \cdot \nabla) \boldsymbol{u})(\boldsymbol{x}) \cdot \boldsymbol{v}(\boldsymbol{x}) \mathrm{d} \boldsymbol{x} .
$$

We now recall the main properties of this problem from [13, Chap. IV, Sect. 2.1] and [23, Chap. 2, Sect. 1], and also the regularity results on its solution from [14, Section 7.3.3] and $[9]$. 
The form $a(\cdot, \cdot)$ is continuous on $H^{1}(\Omega)^{d} \times H^{1}(\Omega)^{d}$ and elliptic on $H_{0}^{1}(\Omega)^{d}$. The form $b(\cdot, \cdot)$ is continuous on $H^{1}(\Omega)^{d} \times L^{2}(\Omega)$ and satisfies the following inf-sup condition, for a constant $\beta_{0}>0$,

$$
\forall q \in L_{\circ}^{2}(\Omega), \quad \sup _{\boldsymbol{v} \in H_{0}^{1}(\Omega)^{d}} \frac{b(\boldsymbol{v}, q)}{\|\boldsymbol{v}\|_{H^{1}(\Omega)^{d}}} \geq \beta_{0}\|q\|_{L^{2}(\Omega)} .
$$

The form $c(\cdot, \cdot, \cdot)$ is continuous on $H^{1}(\Omega)^{d} \times H^{1}(\Omega)^{d} \times H^{1}(\Omega)^{d}$ owing to Sobolev imbeddings and satisfies the following anti-symmetry property, valid for any divergence-free function $\boldsymbol{w}$ in $H^{1}(\Omega)^{d}$,

$$
\forall \boldsymbol{v} \in H_{0}^{1}(\Omega)^{d}, \quad c(\boldsymbol{w}, \boldsymbol{v}, \boldsymbol{v})=0 .
$$

By combining all these properties with Brouwer's fixed-point theorem, the following results can be derived.

Proposition 2.1. (i) For any data $\boldsymbol{f}$ in $H^{-1}(\Omega)^{d}$, problem (2.2) admits at least a solution. Moreover this solution satisfies

$$
\|\boldsymbol{u}\|_{H^{1}(\Omega)^{d}}+\|p\|_{L^{2}(\Omega)} \leq c\|\boldsymbol{f}\|_{H^{-1}(\Omega)^{d}}
$$

where the constant $c$ only depends on $\Omega, \nu$ and $\beta_{0}$.

(ii) If the data $\boldsymbol{f}$ and the viscosity $\nu$ satisfy

$$
\frac{\mathcal{N}}{\nu^{2}}\|\boldsymbol{f}\|_{H^{-1}(\Omega)^{d}}<1
$$

where $\mathcal{N}$ is the norm of $c(\cdot, \cdot, \cdot)$, this solution is unique.

(iii) If the data $\boldsymbol{f}$ belong to $L^{2}(\Omega)^{d}$, the solution $(\boldsymbol{u}, p)$ belongs to $H^{s+1}(\Omega)^{d} \times H^{s}(\Omega)$ for all $s \leq s_{0}$, with $\frac{1}{2} \leq s_{0} \leq 1$ in the general case, $\frac{1}{2}<s_{0} \leq 1$ when $\Omega$ is a polygon or a polyhedron, and $s_{0}=1$ when $\Omega$ is convex.

\subsection{The discrete problem.}

From now on, we assume that $\Omega$ is a polygon $(d=2)$ or a polyhedron $(d=3)$. We introduce a regular family $\left(\mathcal{T}_{h}\right)_{h}$ of triangulations of $\Omega$ by triangles or tetrahedra, in the sense that, for each $h$ :

- $\bar{\Omega}$ is the union of all elements of $\mathcal{T}_{h}$;

- The intersection of two different elements of $\mathcal{T}_{h}$, if not empty, is a vertex or a whole edge or a whole face of both of them;

- The ratio of the diameter $h_{K}$ of any element $K$ of $\mathcal{T}_{h}$ to the diameter of its inscribed circle or sphere is smaller than a constant $\sigma$ independent of $h$.

As usual, $h$ stands for the maximum of the diameters $h_{K}, K \in \mathcal{T}_{h}$.

For each $h$, we introduce a pair of finite element spaces $\mathbb{X}_{h}$ and $\mathbb{M}_{h}$ associated with the triangulation $\mathcal{T}_{h}$ on which we make the following assumption, in order to work with a conforming discretization

$$
\mathbb{X}_{h} \subset H_{0}^{1}(\Omega)^{d} \quad \text { and } \quad \mathbb{M}_{h} \subset L_{\circ}^{2}(\Omega) .
$$


In view of a variational multi-scale discretization, we also consider another pair of finite element spaces $\mathbb{X}_{h^{\prime}}$ and $\mathbb{M}_{h^{\prime}}$ which will be finer than the previous ones, in a sense which is made precise later on. There also, we assume that

$$
\mathbb{X}_{h^{\prime}} \subset H_{0}^{1}(\Omega)^{d} \quad \text { and } \quad \mathbb{M}_{h^{\prime}} \subset L_{\circ}^{2}(\Omega) .
$$

We make the further assumption that both intersections $\mathbb{X}_{h} \cap \mathbb{X}_{h^{\prime}}$ and $\mathbb{M}_{h} \cap \mathbb{M}_{h^{\prime}}$ are reduced to $\{0\}$ and set

$$
\overline{\mathbb{X}}_{h}=\mathbb{X}_{h} \oplus \mathbb{X}_{h^{\prime}} \quad \text { and } \quad \overline{\mathbb{M}}_{h}=\mathbb{M}_{h} \oplus \mathbb{M}_{h^{\prime}}
$$

Remark 2.2. Most often, the spaces $\overline{\mathbb{X}}_{h}$ and $\overline{\mathbb{M}}_{h}$ are constructed from $\mathbb{X}_{h}$ and $\mathbb{M}_{h}$ in one of the following ways:

(i) They are built with polynomials of the same degree as $\mathbb{X}_{h}$ and $\mathbb{M}_{h}$ but associated with a triangulation $\mathcal{T}_{h^{\prime}}$ constructed from $\mathcal{T}_{h}$ by a refinement;

(ii) They are still associated with the triangulation $\mathcal{T}_{h}$ but higher degrees of polynomials are used on each $K$ in $\mathcal{T}_{h}$.

In both cases, there is not a unique way to build the spaces $\mathbb{X}_{h^{\prime}}$ and $\mathbb{M}_{h^{\prime}}$ to have (2.8). Space $\mathbb{X}_{h^{\prime}}$, for instance, may be constructed by means of a surjective linear operator of restriction or interpolation $\Pi_{h}: \overline{\mathbb{X}}_{h} \rightarrow \mathbb{X}_{h}$, by

$$
\mathbb{X}_{h^{\prime}}=\left(\operatorname{Id}-\Pi_{h}\right) \overline{\mathbb{X}}_{h}
$$

where Id is the identity operator. Space $\mathbb{M}_{h^{\prime}}$ is built from $\overline{\mathbb{M}}_{h}$ similarly. In this way $\mathbb{X}_{h^{\prime}}$ and $\mathbb{M}_{h^{\prime}}$ do not need to be explicitly constructed.

We model the eddy viscosity by means of the Smagorinsky model: We associate with each function $\boldsymbol{v}$ in $H_{0}^{1}(\Omega)^{d}$ its eddy viscosity $\nu_{S}(\boldsymbol{v})$ defined by

$$
\forall K \in \mathcal{T}_{h},\left.\quad \nu_{S}(\boldsymbol{v})\right|_{K}=\left(C_{S} h_{K}\right)^{2}|\operatorname{grad} \boldsymbol{v}|,
$$

where $|\cdot|$ here denotes the Euclidean norm on $\mathbb{R}^{d \times d}$. The quantity $C_{S}$ is called Smagorinsky constant, and several values of it have been proposed. It is typically equal to 0.18 , (see Germano [11], [12]) although it can be dynamically adapted in a time-dependent computation (see Lilly [20]). It can also be adjusted close to solid walls, in order to avoid over-diffusion (see Van Driest [24]).

In the sequel we shall assume that the interpolation operator $\Pi_{h}$ is defined from $H_{0}^{1}(\Omega)^{d}$ onto $\mathbb{X}_{h}$ and satisfies the following stability property:

$$
\forall \boldsymbol{v} \in H_{0}^{1}(\Omega)^{d}, \quad\left\|\Pi_{h} \boldsymbol{v}\right\|_{H^{1}(\Omega)^{d}} \leq c\|\boldsymbol{v}\|_{H^{1}(\Omega)^{d}} .
$$

We are thus in a position to write the discrete problem. It reads:

Find $\left(\overline{\boldsymbol{u}}_{h}, \bar{p}_{h}\right)$ in $\overline{\mathbb{X}}_{h} \times \overline{\mathbb{M}}_{h}$ such that

$$
\begin{array}{ll}
\forall \overline{\boldsymbol{v}}_{h} \in \overline{\mathbb{X}}_{h}, \quad a\left(\overline{\boldsymbol{u}}_{h}, \overline{\boldsymbol{v}}_{h}\right)+c\left(\overline{\boldsymbol{u}}_{h}, \overline{\boldsymbol{u}}_{h}, \overline{\boldsymbol{v}}_{h}\right)+b\left(\overline{\boldsymbol{v}}_{h}, \bar{p}_{h}\right) & +a_{S}\left(\overline{\boldsymbol{u}}_{h}, \overline{\boldsymbol{v}}_{h}\right)=\left\langle\boldsymbol{f}, \overline{\boldsymbol{v}}_{h}\right\rangle, \\
\forall \bar{q}_{h} \in \overline{\mathbb{M}}_{h}, \quad b\left(\overline{\boldsymbol{u}}_{h}, \bar{q}_{h}\right)=0, &
\end{array}
$$


where the "Smagorinsky" eddy viscosity form $a_{S}(\cdot, \cdot)$ is now defined by

$$
\begin{array}{r}
a_{S}(\boldsymbol{u}, \boldsymbol{v})=\int_{\Omega} \nu_{S}\left(\boldsymbol{u}_{h^{\prime}}\right)(\boldsymbol{x})\left(\operatorname{grad} \boldsymbol{u}_{h^{\prime}}\right)(\boldsymbol{x}):\left(\operatorname{grad} \boldsymbol{v}_{h^{\prime}}\right)(\boldsymbol{x}) \mathrm{d} \boldsymbol{x} \\
\text { with } \quad \boldsymbol{u}_{h^{\prime}}=\left(\mathrm{Id}-\Pi_{h}\right) \boldsymbol{u}, \boldsymbol{v}_{h^{\prime}}=\left(\mathrm{Id}-\Pi_{h}\right) \boldsymbol{v} .
\end{array}
$$

It can be noted that, up to the eddy viscosity term $a_{S}\left(\overline{\boldsymbol{u}}_{h}, \overline{\boldsymbol{v}}_{h}\right)$, this problem is constructed from (2.2) by the Galerkin method. This term models the sub-grid eddy viscosity effects, that are taken into account by means of the Smagorinsky model with a projection term $\left(\mathrm{Id}-\Pi_{h}\right)$ that filters out the action of eddy viscosity on the large resolved scales.

Remark 2.3. The standard Smagorinsky model models the eddy viscosity effects by the form $\widetilde{a_{S}}$ defined by

$$
\widetilde{a_{S}}(\boldsymbol{u}, \boldsymbol{v})=\int_{\Omega} \nu_{S}(\boldsymbol{u})(\boldsymbol{x})(\operatorname{grad} \boldsymbol{u})(\boldsymbol{x}):(\operatorname{grad} \boldsymbol{v})(\boldsymbol{x}) \mathrm{d} \boldsymbol{x} .
$$

This form $\widetilde{a_{S}}$ does not include the projection term, so the eddy viscosity acts on both large and small resolved scales. This produces an over-diffusive effect, that the projection term intends to correct.

Remark 2.4. Let us define the residual of the Navier-Stokes equations by duality: For any triplet $(\overline{\boldsymbol{v}} ; \boldsymbol{v}, q)$ in $H^{1}(\Omega)^{d} \times H^{1}(\Omega)^{d} \times L^{2}(\Omega)$, the quantity $R(\overline{\boldsymbol{v}} ; \boldsymbol{v}, q)$ belongs to $H^{-1}(\Omega)^{d}$ and satisfies

$$
\forall \boldsymbol{w} \in H_{0}^{1}(\Omega)^{d}, \quad\langle R(\overline{\boldsymbol{v}} ; \boldsymbol{v}, q), \boldsymbol{w}\rangle=a(\boldsymbol{v}, \boldsymbol{w})+c(\overline{\boldsymbol{v}}, \boldsymbol{v}, \boldsymbol{w})+b(\boldsymbol{w}, q)-\langle\boldsymbol{f}, \boldsymbol{w}\rangle,
$$

Then, problem (2.11) is equivalent to the following variational multi-scale method

Find $\left(\overline{\boldsymbol{u}}_{h}, \bar{p}_{h}\right)$ satisfying

$$
\overline{\boldsymbol{u}}_{h}=\boldsymbol{u}_{h}+\boldsymbol{u}_{h^{\prime}}, \quad \bar{p}_{h}=p_{h}+p_{h^{\prime}},
$$

where the pair $\left(\boldsymbol{u}_{h}, p_{h}\right)$ is a solution of the problem

Find $\left(\boldsymbol{u}_{h}, p_{h}\right)$ in $\mathbb{X}_{h} \times \mathbb{M}_{h}$ such that

$$
\begin{array}{ll}
\forall \boldsymbol{v}_{h} \in \mathbb{X}_{h}, \quad a\left(\boldsymbol{u}_{h}, \boldsymbol{v}_{h}\right)+c\left(\overline{\boldsymbol{u}}_{h}, \boldsymbol{u}_{h}, \boldsymbol{v}_{h}\right)+b\left(\boldsymbol{v}_{h}, p_{h}\right)=-\left\langle R\left(\overline{\boldsymbol{u}}_{h} ; \boldsymbol{u}_{h^{\prime}}, p_{h^{\prime}}\right), \boldsymbol{v}_{h}\right\rangle, \\
\forall q_{h} \in \mathbb{M}_{h}, \quad b\left(\boldsymbol{u}_{h}, q_{h}\right)=-b\left(\boldsymbol{u}_{h^{\prime}}, q_{h}\right),
\end{array}
$$

and the pair $\left(\boldsymbol{u}_{h^{\prime}}, p_{h^{\prime}}\right)$ is a solution of the problem

Find $\left(\boldsymbol{u}_{h^{\prime}}, p_{h^{\prime}}\right)$ in $\mathbb{X}_{h^{\prime}} \times \mathbb{M}_{h^{\prime}}$ such that

$$
\begin{aligned}
& \forall \boldsymbol{v}_{h^{\prime}} \in \mathbb{X}_{h^{\prime}}, \quad a\left(\boldsymbol{u}_{h^{\prime}}, \boldsymbol{v}_{h^{\prime}}\right)+c\left(\overline{\boldsymbol{u}}_{h}, \boldsymbol{u}_{h^{\prime}}, \boldsymbol{v}_{h^{\prime}}\right)+b\left(\boldsymbol{v}_{h^{\prime}}, p_{h^{\prime}}\right) \\
& +a_{S}\left(\boldsymbol{u}_{h^{\prime}}, \boldsymbol{v}_{h^{\prime}}\right)=-\left\langle R\left(\overline{\boldsymbol{u}}_{h} ; \boldsymbol{u}_{h}, p_{h}\right), \boldsymbol{v}_{h^{\prime}}\right\rangle, \\
& \forall q_{h^{\prime}} \in \mathbb{M}_{h^{\prime}}, \quad b\left(\boldsymbol{u}_{h^{\prime}}, q_{h^{\prime}}\right)=-b\left(\boldsymbol{u}_{h}, q_{h^{\prime}}\right) .
\end{aligned}
$$


Indeed, observe that, when taking $\overline{\boldsymbol{v}}_{h}$ equal to $\boldsymbol{v}_{h}\left(\right.$ in $\left.\mathbb{X}_{h}\right)$ in $(2.11)$, the term $a_{S}\left(\overline{\boldsymbol{u}}_{h}, \overline{\boldsymbol{v}}_{h}\right)$ vanishes. It follows that if $\left(\overline{\boldsymbol{u}}_{h}, \bar{p}_{h}\right)$ satisfies $(2.11)$, then $\left(\boldsymbol{u}_{h}, p_{h}\right)$ satisfies (2.15). Also, (2.16) follows from (2.11) by taking $\overline{\boldsymbol{v}}_{h}=\boldsymbol{v}_{h^{\prime}}$ and $\bar{p}_{h}=p_{h^{\prime}}$ as test functions. Finally (2.11) follows from (2.15)-(2.16) by summing up these equations, and using that from (2.14), $\boldsymbol{u}_{h^{\prime}}=\left(\mathrm{Id}-\Pi_{h}\right) \overline{\boldsymbol{u}}_{h^{\prime}}$.

This new formulation brings to light the fact that the Smagorinsky eddy viscosity term only acts on the small scales of the discretization. Moreover, that method (2.11) is a method with two grids, but it only needs a grid and an interpolation operator on a virtual coarser grid to be programmed. In fact, method (2.11) includes three grid levels: Large resolved scales (those of $\mathbb{X}_{h}$ ), small resolved scales (those of $\mathbb{X}_{h^{\prime}}$ ) and un-resolved scales (the remaining scales, that are taken into account by means of the eddy diffusion term).

There also, the existence of a solution to problem (2.11) can be derived by using Brouwer's fixed point theorem. However we prefer to postpone this proof to the next section where a more precise result is established. 


\section{A priori analysis.}

As now standard for nonlinear problems, the a priori analysis of the discrete problem (2.11) is performed thanks to the discrete implicit function theorem due to Brezzi, Rappaz and Raviart [7]. This requires some further notation that we now introduce.

We are led to make two further hypotheses:

(i) There exists a constant $\bar{\beta}$ independent of $h$ such that

$$
\forall \bar{q}_{h} \in \overline{\mathbb{M}}_{h}, \quad \sup _{\overline{\boldsymbol{v}}_{h} \in \overline{\mathbb{X}}_{h}} \frac{b\left(\overline{\boldsymbol{v}}_{h}, \bar{q}_{h}\right)}{\left\|\overline{\boldsymbol{v}}_{h}\right\|_{H^{1}(\Omega)^{d}}} \geq \bar{\beta}\left\|\bar{q}_{h}\right\|_{L^{2}(\Omega)} .
$$

(ii) For each nonnegative integer $k$, let $\mathcal{P}_{k}(K)$ denote the space of restrictions to $K$ of polynomials with $d$ variables and total degree $\leq k$. Then, $\overline{\mathbb{X}}_{h}$ contains the space

$$
\mathbb{X}_{h}^{1}=\left\{\boldsymbol{v}_{h} \in H_{0}^{1}(\Omega)^{d} ; \forall K \in \mathcal{T}_{h},\left.v_{h}\right|_{K} \in \mathcal{P}_{1}(K)^{d}\right\},
$$

and $\overline{\mathbb{M}}_{h}$ contains either the space

$$
\mathbb{M}_{h}^{1}=\left\{q_{h} \in H^{1}(\Omega) \cap L_{\circ}^{2}(\Omega) ; \forall K \in \mathcal{T}_{h},\left.q_{h}\right|_{K} \in \mathcal{P}_{1}(K)\right\}
$$

or the space

$$
\mathbb{M}_{h}^{0}=\left\{q_{h} \in L_{\circ}^{2}(\Omega) ; \forall K \in \mathcal{T}_{h},\left.q_{h}\right|_{K} \in \mathcal{P}_{0}(K)\right\} .
$$

This last assumption is satisfied by all the finite element spaces we work with.

In what follows, $c, c^{\prime}, \ldots$ stand for generic constants that may vary from line to line, but are always independent of $h$.

\subsection{Some notation.}

Let $\mathcal{S}$ denote the Stokes operator, more precisely the operator which associates with any data $\boldsymbol{f}$ in $H^{-1}(\Omega)^{d}$ the part $\boldsymbol{u}$ of the solution $(\boldsymbol{u}, p)$ of the problem

Find $(\boldsymbol{u}, p)$ in $H_{0}^{1}(\Omega)^{d} \times L_{\circ}^{2}(\Omega)$ such that

$$
\begin{aligned}
& \forall \boldsymbol{v} \in H_{0}^{1}(\Omega)^{d}, \quad a(\boldsymbol{u}, \boldsymbol{v})+b(\boldsymbol{v}, p)=\langle\boldsymbol{f}, \boldsymbol{v}\rangle, \\
& \forall q \in L_{\circ}^{2}(\Omega), \quad b(\boldsymbol{u}, q)=0 .
\end{aligned}
$$

It follows from the properties of the forms $a(\cdot, \cdot)$ and $b(\cdot, \cdot)$ stated in Section 2.1 that this problem is well-posed. So, the operator $\mathcal{S}$ is well defined and continuous from $H^{-1}(\Omega)^{d}$ into $H_{0}^{1}(\Omega)^{d}$. Owing to the inf-sup condition $(2.3)$, it is readily checked that problem $(2.2)$ can equivalently be written as a fixed-point equation

$$
\mathcal{F}(\boldsymbol{u})=\boldsymbol{u}-\mathcal{S G}(\boldsymbol{u})=0, \quad \text { with } \quad \mathcal{G}(\boldsymbol{u})=\boldsymbol{f}-(\boldsymbol{u} \cdot \nabla) \boldsymbol{u} .
$$

Similarly, let $\overline{\mathcal{S}}_{h}$ be the discrete Stokes operator, i.e., the operator which associates with any data $\boldsymbol{f}$ in $H^{-1}(\Omega)^{d}$ the part $\overline{\boldsymbol{u}}_{h}$ of the solution $\left(\overline{\boldsymbol{u}}_{h}, \overline{\boldsymbol{p}}_{h}\right)$ of the problem 
Find $\left(\overline{\boldsymbol{u}}_{h}, \bar{p}_{h}\right)$ in $\overline{\mathbb{X}}_{h} \times \bar{M}_{h}$ such that

$$
\begin{array}{ll}
\forall \overline{\boldsymbol{v}}_{h} \in \overline{\mathbb{X}}_{h}, \quad a\left(\overline{\boldsymbol{u}}_{h}, \overline{\boldsymbol{v}}_{h}\right)+b\left(\overline{\boldsymbol{v}}_{h}, \bar{p}_{h}\right)=\left\langle\boldsymbol{f}, \overline{\boldsymbol{v}}_{h}\right\rangle, \\
\forall \bar{q}_{h} \in \overline{\mathbb{M}}_{h}, \quad b\left(\overline{\boldsymbol{u}}_{h}, \bar{q}_{h}\right)=0 .
\end{array}
$$

There also, owing to (3.1) this operator is well-defined.

Let us now introduce the mapping associated with the Smagorinsky form: It is defined from $H_{0}^{1}(\Omega)^{d}$ into $H^{-1}(\Omega)^{d}$ by

$$
\forall \boldsymbol{u} \in H_{0}^{1}(\Omega)^{d}, \forall \boldsymbol{v} \in H_{0}^{1}(\Omega)^{d}, \quad\left\langle\mathcal{A}_{S}(\boldsymbol{u}), \boldsymbol{v}\right\rangle=a_{S}(\boldsymbol{u}, \boldsymbol{v}) .
$$

Thus, problem (2.11) can equivalently be written

$$
\mathcal{F}_{h}\left(\overline{\boldsymbol{u}}_{h}\right)=\overline{\boldsymbol{u}}_{h}-\overline{\mathcal{S}}_{h} \overline{\mathcal{G}}_{h}\left(\overline{\boldsymbol{u}}_{h}\right)=0, \quad \text { with } \quad \overline{\mathcal{G}}_{h}(\boldsymbol{u})=\mathcal{G}(\boldsymbol{u})-\mathcal{A}_{S}(\boldsymbol{u})
$$

To go further, we need some properties of the operator $\overline{\mathcal{S}}_{h}$.

\subsection{Basic properties of the discrete Stokes operator.}

The stability property of the operator $\overline{\mathcal{S}}_{h}$ is immediately derived by taking $\overline{\boldsymbol{v}}_{h}$ equal to $\overline{\boldsymbol{u}}_{h}$ in problem (3.7).

Lemma 3.1. The operator $\overline{\mathcal{S}}_{h}$ satisfies the following stability property: For all $\boldsymbol{f}$ in $H^{-1}(\Omega)^{d}$,

$$
\left\|\overline{\mathcal{S}}_{h} \boldsymbol{f}\right\|_{H^{1}(\Omega)^{d}} \leq c \sup _{\overline{\boldsymbol{v}}_{h} \in \overline{\mathbb{X}}_{h}} \frac{\left\langle\boldsymbol{f}, \overline{\boldsymbol{v}}_{h}\right\rangle}{\left\|\overline{\boldsymbol{v}}_{h}\right\|_{H^{1}(\Omega)^{d}}} .
$$

We refer to $[6, \S I V .2]$ and [13, Chap. II] among others for the following convergence properties which require the assumptions on $\overline{\mathbb{X}}_{h}$ and $\overline{\mathbb{M}}_{h}$ made in the beginning of the section.

Lemma 3.2. The operator $\overline{\mathcal{S}}_{h}$ satisfies the following convergence property: For all $\boldsymbol{f}$ in $L^{2}(\Omega)^{d}$,

$$
\left\|\left(\mathcal{S}-\overline{\mathcal{S}}_{h}\right) \boldsymbol{f}\right\|_{H^{1}(\Omega)^{d}} \leq c h^{s_{0}}\|\boldsymbol{f}\|_{L^{2}(\Omega)^{d}}
$$

where the real number $s_{0}$ is introduced in Proposition 2.1 .

From Lemmas 3.1 and 3.2, we easily derive that, for all $\boldsymbol{f}$ in $H^{-1}(\Omega)^{d}$,

$$
\lim _{h \rightarrow 0}\left\|\left(\mathcal{S}-\overline{\mathcal{S}}_{h}\right) \boldsymbol{f}\right\|_{H^{1}(\Omega)^{d}}=0,
$$

which will be of great use in what follows.

\subsection{Preliminary lemmas.}


From now on, we assume that $\boldsymbol{f}$ belongs to $L^{2}(\Omega)^{d}$ and that $(\boldsymbol{u}, p)$ is a nonsingular solution of problem (2.2), in the sense made precise in [7], see also [13, Chap. IV, Sect. 3.1]: The operator $D \mathcal{F}(\boldsymbol{u})=\operatorname{Id}-\mathcal{S} D \mathcal{G}(\boldsymbol{u})$ is an isomorphism of $H_{0}^{1}(\Omega)^{d}$ (where $D$ denotes the differential operator with respect to $\boldsymbol{u})$. It can be noted that this assumption is much less restrictive than the global uniqueness of the solution (see part (ii) of Proposition 2.1) since it only ensures its local uniqueness.

We denote by $\mathcal{L}(E, F)$ the space of linear continuous mappings from a normed space $E$ on another normed space $F$ and by $\mathcal{E}$ the space of endomorphisms of $H_{0}^{1}(\Omega)^{d}: \mathcal{E}=$ $\mathcal{L}\left(H_{0}^{1}(\Omega)^{d}, H_{0}^{1}(\Omega)^{d}\right)$.

Lemma 3.3. If property (2.10) holds, there exists an $h_{0}>0$ such that, for all $h \leq h_{0}$, the operator $D \mathcal{F}_{h}(\boldsymbol{u})$ is an isomorphism of $H_{0}^{1}(\Omega)^{d}$ and the norm $\mu$ of its inverse is bounded independently of $h$.

Proof: We have

$$
D \mathcal{F}_{h}(\boldsymbol{u})=D \mathcal{F}(\boldsymbol{u})+\left(\mathcal{S}-\overline{\mathcal{S}}_{h}\right) D \mathcal{G}(\boldsymbol{u})+\overline{\mathcal{S}}_{h}\left(D \mathcal{G}(\boldsymbol{u})-D \overline{\mathcal{G}}_{h}(\boldsymbol{u})\right)
$$

Since $D \mathcal{F}(\boldsymbol{u})$ is an isomorphism of $H_{0}^{1}(\Omega)^{d}$, the desired property will be established if the last two terms in the previous expansion tend to zero. We study successively these two terms.

1) We have

$$
D \mathcal{G}(\boldsymbol{u}) \cdot \boldsymbol{w}=-(\boldsymbol{u} \cdot \nabla) \boldsymbol{w}-(\boldsymbol{w} \cdot \nabla) \boldsymbol{u} .
$$

Since $s_{0}>\frac{1}{2}$, see Proposition 2.1, the mapping: $\boldsymbol{w} \mapsto D \mathcal{G}(\boldsymbol{u}) \cdot \boldsymbol{w}$ sends the unit sphere of $H_{0}^{1}(\Omega)^{d}$ into a compact subset of $H^{-1}(\Omega)^{d}$. Thus, since for all $\varepsilon>0$, a compact subset admits an overlap by a finite number of balls with centre $f_{i}$ and radius $\varepsilon$, applying (3.12) to all these $f_{i}$ yields that

$$
\lim _{h \rightarrow 0}\left\|\left(\mathcal{S}-\overline{\mathcal{S}}_{h}\right) D \mathcal{G}(\boldsymbol{u})\right\|_{\mathcal{E}}=0 .
$$

2) Since $D \mathcal{G}(\boldsymbol{u})-D \overline{\mathcal{G}}_{h}(\boldsymbol{u})$ is equal to $D \mathcal{A}_{S}(\boldsymbol{u})$ and thanks to (3.10), we have to evaluate the quantity, for $\boldsymbol{w}$ running through the unit ball of $H_{0}^{1}(\Omega)^{d}$ and $\overline{\boldsymbol{z}}_{h}$ running through the unit ball of $\overline{\mathbb{X}}_{h}$,

$$
\begin{aligned}
\mathcal{B}_{S}\left(\boldsymbol{u} ; \boldsymbol{w}, \overline{\boldsymbol{z}}_{h}\right)=C_{S}^{2} \sum_{K \in \mathcal{T}_{h}} h_{K}^{2}\left(\int_{K}\left|\operatorname{grad} \boldsymbol{u}^{*}\right|(\boldsymbol{x})\left(\operatorname{grad} \boldsymbol{w}^{*}\right)(\boldsymbol{x}):\left(\operatorname{grad} \overline{\boldsymbol{z}}_{h}^{*}\right)(\boldsymbol{x}) \mathrm{d} \boldsymbol{x}\right. \\
\left.+\int_{K}\left(\frac{\operatorname{grad} \boldsymbol{u}^{*}: \operatorname{grad} \boldsymbol{w}^{*}}{\left|\operatorname{grad} \boldsymbol{u}^{*}\right|}\right)(\boldsymbol{x})\left(\operatorname{grad} \boldsymbol{u}^{*}\right)(\boldsymbol{x}):\left(\operatorname{grad} \overline{\boldsymbol{z}}_{h}^{*}\right)(\boldsymbol{x}) \mathrm{d} \boldsymbol{x}\right),
\end{aligned}
$$

where for brevity, for any function $\boldsymbol{v}$, we denote by $\boldsymbol{v}^{*}$ the function $\left(\operatorname{Id}-\Pi_{h}\right) \boldsymbol{v}$. By using Cauchy-Schwarz inequalities and also property (2.10), we obtain

$$
\left|\mathcal{B}_{S}\left(\boldsymbol{u} ; \boldsymbol{w}, \overline{\boldsymbol{z}}_{h}\right)\right| \leq C_{S}^{2} \sum_{K \in \mathcal{T}_{h}} h_{K}^{2}\|\operatorname{grad} \boldsymbol{u}\|_{L^{2}(K)^{d \times d}}\|\operatorname{grad} \boldsymbol{w}\|_{L^{2}(K)^{d \times d}}\left\|\operatorname{grad} \overline{\boldsymbol{z}}_{h}\right\|_{L^{\infty}(K)^{d \times d}}
$$

We recall from [4, Chap. VII, Prop. 4.2] the local inverse inequality, valid for any polynomial $\varphi$ of fixed degree,

$$
\|\varphi\|_{L^{\infty}(K)} \leq c h_{K}^{-\frac{d}{2}}\|\varphi\|_{L^{2}(K)} .
$$


Applying it to each component of $\operatorname{grad} \overline{\boldsymbol{z}}_{h}$ yields

$$
\left|\mathcal{B}_{S}\left(\boldsymbol{u} ; \boldsymbol{w}, \overline{\boldsymbol{z}}_{h}\right)\right| \leq c h^{2-\frac{d}{2}}\|\boldsymbol{u}\|_{H^{1}(\Omega)^{d}}\|\boldsymbol{w}\|_{H^{1}(\Omega)^{d}}\left\|\boldsymbol{z}_{h}\right\|_{H^{1}(\Omega)^{d}},
$$

whence

$$
\left\|D \mathcal{G}(\boldsymbol{u})-D \overline{\mathcal{G}}_{h}(\boldsymbol{u})\right\|_{\mathcal{L}\left(H_{0}^{1}(\Omega)^{d}, H^{-1}(\Omega)^{d}\right)} \leq c h^{2-\frac{d}{2}}\|\boldsymbol{u}\|_{H^{1}(\Omega)^{d}}
$$

and

$$
\lim _{h \rightarrow 0}\left\|\overline{\mathcal{S}}_{h}\left(D \mathcal{G}(\boldsymbol{u})-D \mathcal{G}_{h}(\boldsymbol{u})\right)\right\|_{\mathcal{E}}=0
$$

This concludes the proof of the lemma.

Lemma 3.4. If property (2.10) holds, there exists a neighbourhood of $\boldsymbol{u}$ and a constant $\lambda>0$ independent of $h$ such that the operator $D \mathcal{F}_{h}$ satisfies the following Lipschitz property for any $\boldsymbol{v}$ in this neighbourhood

$$
\left\|D \mathcal{F}_{h}(\boldsymbol{u})-D \mathcal{F}_{h}(\boldsymbol{v})\right\|_{\mathcal{E}} \leq \lambda\|\boldsymbol{u}-\boldsymbol{v}\|_{H^{1}(\Omega)^{d}} .
$$

Proof: Using $\overline{\mathcal{G}}=\mathcal{G}-\mathcal{A}_{S}$ we write

$$
D \mathcal{F}_{h}(\boldsymbol{u})-D \mathcal{F}_{h}(\boldsymbol{v})=-\overline{\mathcal{S}}_{h}(D \mathcal{G}(\boldsymbol{u})-D \mathcal{G}(\boldsymbol{v}))-\overline{\mathcal{S}}_{h}\left(D \mathcal{A}_{S}(\boldsymbol{u})-D \mathcal{A}_{S}(\boldsymbol{v})\right)
$$

Bounding the first term readily follows from Lemma 3.1 and (3.13). To estimate the second one, thanks to Lemma 3.1 and with the same notation as in the previous proof, we have to bound the quantities, for $\boldsymbol{w}$ running through the unit ball of $H_{0}^{1}(\Omega)^{d}$ and $\overline{\boldsymbol{z}}_{h}$ running through the unit ball of $\overline{\mathbb{X}}_{h}$,

$$
\begin{aligned}
& \mathcal{B}_{S}\left(\boldsymbol{u} ; \boldsymbol{w}, \overline{\boldsymbol{z}}_{h}\right)-\mathcal{B}_{S}\left(\boldsymbol{v} ; \boldsymbol{w}, \overline{\boldsymbol{z}}_{h}\right) \\
&=C_{S}^{2} \sum_{K \in \mathcal{T}_{h}} h_{K}^{2}\left(\int_{K}\left(\left|\operatorname{grad} \boldsymbol{u}^{*}\right|-\left|\operatorname{grad} \boldsymbol{v}^{*}\right|\right) \mid(\boldsymbol{x})\left(\operatorname{grad} \boldsymbol{w}^{*}\right)(\boldsymbol{x}):\left(\operatorname{grad} \bar{z}_{h}^{*}\right)(\boldsymbol{x}) \mathrm{d} \boldsymbol{x}\right. \\
&+\int_{K}\left(\frac{\operatorname{grad} \boldsymbol{u}^{*}: \operatorname{grad} \boldsymbol{w}^{*}}{\left|\operatorname{grad} \boldsymbol{u}^{*}\right|}\right)(\boldsymbol{x})\left(\operatorname{grad} \boldsymbol{u}^{*}\right)(\boldsymbol{x}):\left(\operatorname{grad} \overline{\boldsymbol{z}}_{h}^{*}\right)(\boldsymbol{x}) \mathrm{d} \boldsymbol{x} \\
&\left.\quad-\int_{K}\left(\frac{\operatorname{grad} \boldsymbol{v}^{*}: \operatorname{grad} \boldsymbol{w}^{*}}{\left|\operatorname{grad} \boldsymbol{v}^{*}\right|}\right)(\boldsymbol{x})\left(\operatorname{grad} \boldsymbol{v}^{*}\right)(\boldsymbol{x}):\left(\operatorname{grad} \overline{\boldsymbol{z}}_{h}^{*}\right)(\boldsymbol{x}) \mathrm{d} \boldsymbol{x}\right) .
\end{aligned}
$$

The inverse inequatity (3.15) yields the estimate for the first term. To handle the remainder, that we denote by $\mathcal{D}_{S}$ for brevity, we first use a triangle inequality, next add and subtract a further term.

$$
\begin{aligned}
& \mathcal{D}_{S}= C_{S}^{2} \sum_{K \in \mathcal{T}_{h}} h_{K}^{2}\left(\int_{K}\left(\frac{\operatorname{grad} \boldsymbol{u}^{*}: \operatorname{grad} \boldsymbol{w}^{*}}{\left|\operatorname{grad} \boldsymbol{u}^{*}\right|}\right)(\boldsymbol{x})\left(\operatorname{grad}\left(\boldsymbol{u}^{*}-\boldsymbol{v}^{*}\right)\right)(\boldsymbol{x}):\left(\operatorname{grad} \overline{\boldsymbol{z}}_{h}^{*}\right)(\boldsymbol{x}) \mathrm{d} \boldsymbol{x}\right. \\
&+\int_{K}\left(\frac{\left(\left|\operatorname{grad} \boldsymbol{v}^{*}\right|-\left|\operatorname{grad} \boldsymbol{u}^{*}\right|\right) \operatorname{grad} \boldsymbol{u}^{*}: \operatorname{grad} \boldsymbol{w}^{*}}{\left|\operatorname{grad} \boldsymbol{u}^{*}\right|\left|\operatorname{grad} \boldsymbol{v}^{*}\right|}\right)(\boldsymbol{x})\left(\operatorname{grad} \boldsymbol{v}^{*}\right)(\boldsymbol{x}):\left(\operatorname{grad} \overline{\boldsymbol{z}}_{h}^{*}\right)(\boldsymbol{x}) \mathrm{d} \boldsymbol{x} \\
&\left.\quad+\int_{K}\left(\frac{\left|\operatorname{grad} \boldsymbol{u}^{*}\right| \operatorname{grad}\left(\boldsymbol{u}^{*}-\boldsymbol{v}^{*}\right): \operatorname{grad} \boldsymbol{w}^{*}}{\left|\operatorname{grad} \boldsymbol{u}^{*}\right|\left|\operatorname{grad} \boldsymbol{v}^{*}\right|}\right)(\boldsymbol{x})\left(\operatorname{grad} \boldsymbol{v}^{*}\right)(\boldsymbol{x}):\left(\operatorname{grad} \overline{\boldsymbol{z}}_{h}^{*}\right)(\boldsymbol{x}) \mathrm{d} \boldsymbol{x}\right)
\end{aligned}
$$


There also, we use Cauchy-Schwarz inequalities, note that

$$
|| \operatorname{grad} v^{*}|-| \operatorname{grad} u^{*}|| \leq\left|\operatorname{grad}\left(\boldsymbol{u}^{*}-v^{*}\right)\right|
$$

and conclude by using (3.15).

Lemma 3.5. If property (2.10) is satisfied, the following estimate holds for the quantity $\varepsilon_{h}=\left\|\mathcal{F}_{h}(\boldsymbol{u})\right\|_{H^{1}(\Omega)^{d}}$ :

$$
\varepsilon_{h} \leq c(\boldsymbol{f}) h^{\min \left\{s_{0}, 2-\frac{d}{2}\right\}},
$$

where the real number $s_{0}$ is introduced in Proposition 2.1 and $c(\boldsymbol{f})$ only depends on $\|\boldsymbol{f}\|_{L^{2}(\Omega)^{d}}$.

Proof: We observe that

$$
\left\|\mathcal{F}_{h}(\boldsymbol{u})\right\|_{H^{1}(\Omega)^{d}}=\left\|\mathcal{F}(\boldsymbol{u})-\mathcal{F}_{h}(\boldsymbol{u})\right\|_{H^{1}(\Omega)^{d}} \leq\left\|\left(\mathcal{S}-\overline{\mathcal{S}}_{h}\right) \mathcal{G}(\boldsymbol{u})\right\|_{H^{1}(\Omega)^{d}}+\left\|\overline{\mathcal{S}}_{h} \mathcal{A}_{S}(\boldsymbol{u})\right\|_{H^{1}(\Omega)^{d}}
$$

The first term is bounded by $c(\boldsymbol{f}) h^{s_{0}}$ owing to Lemma 3.2. To estimate the second one, we observe that, for $\overline{\boldsymbol{z}}_{h}$ running through the unit ball of $\overline{\mathbb{X}}_{h}$,

$$
a_{S}\left(\boldsymbol{u}, \overline{\boldsymbol{z}}_{h}\right)=C_{S}^{2} \sum_{K \in \mathcal{T}_{h}} h_{K}^{2} \int_{K}\left|\operatorname{grad} u_{h^{\prime}}\right|(\boldsymbol{x})\left(\operatorname{grad} \boldsymbol{u}_{h^{\prime}}\right)(\boldsymbol{x}):\left(\operatorname{grad} \boldsymbol{z}_{h^{\prime}}\right)(\boldsymbol{x}) \mathrm{d} \boldsymbol{x},
$$

with $u_{h^{\prime}}=\left(\operatorname{Id}-\Pi_{h}\right) \boldsymbol{u}, \boldsymbol{z}_{h^{\prime}}=\left(\operatorname{Id}-\Pi_{h}\right) \overline{\boldsymbol{z}}_{h}$. We use once $(3.15)$ to bound $\left\|\operatorname{grad} \overline{\boldsymbol{z}}_{h}\right\|_{L^{\infty}(\Omega) d \times d}$, which yields a bound by a constant times $h^{2-\frac{2}{d}}$. All this leads to the desired result.

\subsection{A priori error estimates for the discrete problem.}

Thanks to the previous technical lemmas, we are in a position to prove the main result of this section owing to the key Theorem in [7].

Theorem 3.6. Assume that the data $\boldsymbol{f}$ belongs to $L^{2}(\Omega)^{d}$ and that $(\boldsymbol{u}, p)$ is a nonsingular solution of problem (2.2), together with condition (2.10). There exists a neighbourhood of $\boldsymbol{u}$ and a real number $h_{0}^{*}$ such that, for all $h \leq h_{0}^{*}$, problem (2.11) has a unique solution $\left(\overline{\boldsymbol{u}}_{h}, \bar{p}_{h}\right)$ with $\overline{\boldsymbol{u}}_{h}$ in this neighbourhood. Moreover, the following a priori error estimate holds

$$
\left\|\boldsymbol{u}-\overline{\boldsymbol{u}}_{h}\right\|_{H^{1}(\Omega)^{d}}+\left\|p-\bar{p}_{h}\right\|_{L^{2}(\Omega)} \leq c(\boldsymbol{f}) h^{\min \left\{s_{0}, 2-\frac{d}{2}\right\}},
$$

where the real number $s_{0}$ is introduced in Proposition 2.1 and $c(\boldsymbol{f})$ only depends on $\|\boldsymbol{f}\|_{L^{2}(\Omega)^{d}}$.

Proof: Since the quantity $\varepsilon_{h}$ tends to zero when $h$ tends to zero, when taking $h_{0}^{*}$ such that $4 \lambda \mu^{2} \varepsilon_{h_{0}^{*}}<1$, the existence of a $\overline{\boldsymbol{u}}_{h}$ solution of problem (3.9) in the ball with centre $\boldsymbol{u}$ and radius $<\frac{1}{2 \lambda \mu}$ and the estimate for $\left\|\boldsymbol{u}-\overline{\boldsymbol{u}}_{h}\right\|_{H^{1}(\Omega)^{d}}$ are a direct consequence of [7, Thm 1] (see also [13, Chap. IV, Thm 3.1]). Then the existence of a $\bar{p}_{h}$ such that $\left(\overline{\boldsymbol{u}}_{h}, \bar{p}_{h}\right)$ is a solution of problem (2.11) and the estimate for $\left\|p-\bar{p}_{h}\right\|_{L^{2}(\Omega)}$ are easily derived from the inf-sup condition (3.1). 
Of course, higher order estimates can be derived when the solution $(\boldsymbol{u}, p)$ is smoother. From now on, $d_{m}(\boldsymbol{v}, E)$ stands for the distance of a function $\boldsymbol{v}$ to a Banach space $E$ in the norm of $H^{m}(\Omega)$.

Corollary 3.7. Assume that the operator $\Pi_{h}$ satisfies for all $s, 0 \leq s \leq 2$, and for all $\boldsymbol{v}$ in $H^{s+1}(\Omega)^{d}$,

$$
\left\|\left(\mathrm{Id}-\Pi_{h}\right) \boldsymbol{v}\right\|_{H^{1}(\Omega)^{d}} \leq c h^{s}\|\boldsymbol{v}\|_{H^{s+1}(\Omega)^{d}} .
$$

If the Assumptions of Theorem 3.6 hold and moreover the solution $(\boldsymbol{u}, p)$ belongs to the space $H^{s+1}(\Omega)^{d} \times H^{s}(\Omega)$ for some $s>\frac{d}{2}$, the following a priori error estimate holds

$$
\left\|\boldsymbol{u}-\overline{\boldsymbol{u}}_{h}\right\|_{H^{1}(\Omega)^{d}}+\left\|p-\bar{p}_{h}\right\|_{L^{2}(\Omega)} \leq c(\boldsymbol{u}, p)\left(h^{2}+d_{1}\left(\boldsymbol{u}, \overline{\mathbb{X}}_{h}\right)+d_{0}\left(p, \overline{\mathbb{M}}_{h}\right)\right)
$$

where the constant $c(\boldsymbol{u}, p)$ now depends on the norms of $\boldsymbol{u}$ and $p$ in these new spaces.

It can be noted that in any case the convergence order is limited to 2 due to the addition of the subgrid eddy viscosity term. So it is useless to work with very high degree polynomials. In any case, a convergence of order 2 for smooth solutions of Navier-Stokes equations is a good result, we do not intend to go further. 


\section{A posteriori analysis.}

We now introduce the error indicators we work with. We successively prove an upper bound for the error (as a function of the indicators), next upper bounds for the indicators.

\subsection{The error indicators.}

We agree to denote by $\overline{\mathcal{T}}_{h}$ the triangulation $\mathcal{T}_{h}$ if the spaces $\mathbb{X}_{h^{\prime}}$ and $\mathbb{M}_{h^{\prime}}$ are associated with this same triangulation or the triangulation $\mathcal{T}_{h^{\prime}}$ if the spaces $\mathbb{X}_{h^{\prime}}$ and $\mathbb{M}_{h^{\prime}}$ are associated with a refined triangulation $\mathcal{T}_{h^{\prime}}$. For each $K$ in $\overline{\mathcal{T}}_{h}$, we denote by $\mathcal{E}_{K}$ the set of edges $(d=2)$ or faces $(d=3)$ of $K$ which are not contained in $\partial \Omega$. For each $e$ in $\mathcal{E}_{K}, h_{e}$ stands for the length $(d=2)$ or diameter $(d=3)$ of $e$ and the jump through $e$ is denoted by $[\cdot]_{e}$ (we do not make its sign precise since it is not necessary).

From now on, we assume that the data $\boldsymbol{f}$ belong to $L^{2}(\Omega)^{d}$ and we consider an approximation $\boldsymbol{f}_{h}$ of $\boldsymbol{f}$ which is polynomial on each element $K$ of $\overline{\mathcal{T}}_{h}$.

We prefer to introduce two families of error indicators, in order to treat separately the subgrid eddy viscosity term:

(i) for each $K$ in $\overline{\mathcal{T}}_{h}$, the error indicator linked to the variational multi-scale discretization is defined by

$$
\begin{aligned}
\eta_{K}=h_{K} \| \boldsymbol{f}_{h}+\nu \Delta \overline{\boldsymbol{u}}_{h} & -\left(\overline{\boldsymbol{u}}_{h} \cdot \nabla\right) \overline{\boldsymbol{u}}_{h}-\operatorname{grad} \bar{p}_{h} \|_{L^{2}(K)^{d}} \\
& +\sum_{e \in \mathcal{E}_{K}} h_{e}^{\frac{1}{2}}\left\|\left[\nu \partial_{n} \overline{\boldsymbol{u}}_{h}-\bar{p}_{h} \boldsymbol{n}\right]_{e}\right\|_{L^{2}(e)^{d}}+\left\|\operatorname{div} \overline{\boldsymbol{u}}_{h}\right\|_{L^{2}(K)} .
\end{aligned}
$$

(ii) for each $K$ in $\overline{\mathcal{T}}_{h}$, the error indicator linked to the eddy viscosity term is defined by

$$
\eta_{S K}=\left(C_{S} h_{K}\right)^{2}\left\|\operatorname{grad} \boldsymbol{u}_{h^{\prime}}\right\|_{L^{4}(K)^{d \times d}}^{2} .
$$

It can be noted that all these indicators are easy to compute once the discrete solution is kwown.

\subsection{Upper bounds for the error.}

We now compute the residuals of the discrete equation, namely the quantity $\boldsymbol{R}$ defined for any $\boldsymbol{v}$ in $H_{0}^{1}(\Omega)^{d}$ by

$$
\langle\boldsymbol{R}, \boldsymbol{v}\rangle=\langle\boldsymbol{f}, \boldsymbol{v}\rangle-a\left(\overline{\boldsymbol{u}}_{h}, \boldsymbol{v}\right)-c\left(\overline{\boldsymbol{u}}_{h}, \overline{\boldsymbol{u}}_{h}, \boldsymbol{v}\right)-b\left(\boldsymbol{v}, \bar{p}_{h}\right),
$$

and its analogue $R^{*}$ defined for any $q$ in $L^{2}(\Omega)$ by

$$
\int_{\Omega} R^{*}(\boldsymbol{x}) q(\boldsymbol{x}) \mathrm{d} \boldsymbol{x}=\int_{\Omega}\left(\operatorname{div} \overline{\boldsymbol{u}}_{h}\right)(\boldsymbol{x}) q(\boldsymbol{x}) \mathrm{d} \boldsymbol{x} .
$$


By substracting the discrete problem (2.11), we obtain, for all $\overline{\boldsymbol{v}}_{h}$ in $\overline{\mathbb{X}}_{h}$,

$$
\langle\boldsymbol{R}, \boldsymbol{v}\rangle=\left\langle\boldsymbol{f}, \boldsymbol{v}-\overline{\boldsymbol{v}}_{h}\right\rangle-a\left(\overline{\boldsymbol{u}}_{h}, \boldsymbol{v}-\overline{\boldsymbol{v}}_{h}\right)-c\left(\overline{\boldsymbol{u}}_{h}, \overline{\boldsymbol{u}}_{h}, \boldsymbol{v}-\overline{\boldsymbol{v}}_{h}\right)-b\left(\boldsymbol{v}-\overline{\boldsymbol{v}}_{h}, \bar{p}_{h}\right)-a_{S}\left(\overline{\boldsymbol{u}}_{h}, \overline{\boldsymbol{v}}_{h}\right) .
$$

We assume that the operator $\Pi_{h}$ is locally stable: There exists a constant $c$ independent of $h$ such that for all $\boldsymbol{v} \in H^{1}(\Omega)^{d}$, and for each $K \in \overline{\mathcal{T}}_{h}$,

$$
\left\|\Pi_{h} \boldsymbol{v}\right\|_{H^{1}(K)} \leq c\|\boldsymbol{v}\|_{H^{1}\left(\delta_{K}\right)}
$$

where $\delta_{K}$ is a finite set of elements of $\overline{\mathcal{T}}_{h}$, whose cardinal is uniformly bounded in $h$.

This property is verified by interpolation operators that are defined locally. The set $\delta_{K}$ is typically formed by elements of $\overline{\mathcal{T}}_{h}$ located in a neighborhood of $K$ of radius of order $h$ (Cf. [4]).

This leads to the following lemma.

Lemma 4.1. Assume that the space $\overline{\mathbb{X}}_{h}$ contains the space $\mathbb{X}_{h}^{1}$ introduced in (3.2), that the operator $\Pi_{h}$ is locally stable and that property (2.10) holds. Then the residual $\boldsymbol{R}$ satisfies the following estimate

$$
\|\boldsymbol{R}\|_{H^{-1}(\Omega)^{d}} \leq c\left(\sum_{K \in \overline{\mathcal{T}}_{h}}\left(\eta_{K}^{2}+\eta_{S K}^{2}+h_{K}^{2}\left\|\boldsymbol{f}-\boldsymbol{f}_{h}\right\|_{L^{2}(K)^{d}}^{2}\right)\right)^{\frac{1}{2}} .
$$

Proof: This estimate relies on fully standard arguments, more precisely, starting from equation (4.5):

1) We add and subtract the term $\left\langle\boldsymbol{f}_{h}, \boldsymbol{v}-\overline{\boldsymbol{v}}_{h}\right\rangle$;

2) We integrate by parts the terms $a\left(\overline{\boldsymbol{u}}_{h}, \boldsymbol{v}-\overline{\boldsymbol{v}}_{h}\right)$ and $b\left(\overline{\boldsymbol{v}}_{h}, \bar{p}_{h}\right)$ on each $K$;

3) Using the fact that $\overline{\mathbb{X}}_{h}$ contains the space $\mathbb{X}_{h}^{1}$ introduced in (3.2), we take $\overline{\boldsymbol{v}}_{h}$ equal to the image of $\boldsymbol{v}$ by a Clément type regularization operator (see [4, Chap. IX, Section 3] for instance), so that for each $K$ in $\overline{\mathcal{T}}_{h}$ and each $e$ in $\mathcal{E}_{K}$,

$$
\left\|\boldsymbol{v}-\overline{\boldsymbol{v}}_{h}\right\|_{H^{1}(K)^{d}}+h_{e}^{-\frac{1}{2}}\left\|\boldsymbol{v}-\overline{\boldsymbol{v}}_{h}\right\|_{L^{2}(e)^{d}}+h_{K}^{-1}\left\|\boldsymbol{v}-\overline{\boldsymbol{v}}_{h}\right\|_{L^{2}(K)^{d}} \leq c\|\boldsymbol{v}\|_{H^{1}\left(\Delta_{K}\right)^{d}},
$$

where $\Delta_{K}$ is the union of elements of $\overline{\mathcal{T}}_{h}$ which intersect $K$;

4) Finally, we bound $a_{S}\left(\overline{\boldsymbol{u}}_{h}, \overline{\boldsymbol{v}}_{h}\right)$ by

$$
a_{S}\left(\overline{\boldsymbol{u}}_{h}, \overline{\boldsymbol{v}}_{h}\right) \leq \sum_{K \in \overline{\mathcal{T}}_{h}}\left(C_{S} h_{K}\right)^{2}\left\|\nabla \boldsymbol{u}_{h^{\prime}}\right\|_{L^{4}(K)^{d \times d}}^{2}\left\|\nabla \boldsymbol{v}_{h^{\prime}}\right\|_{L^{2}(K)^{d \times d}}
$$

Using the local stability of $\Pi_{h}$, since $\boldsymbol{v}_{h^{\prime}}$ is equal to $\left(\mathrm{Id}-\Pi_{h}\right) \overline{\boldsymbol{v}}_{h}$,

$$
\left\|\boldsymbol{v}_{h^{\prime}}\right\|_{H^{1}(K)^{d}} \leq c\left\|\overline{\boldsymbol{v}}_{h}\right\|_{H^{1}\left(\delta_{K}\right)^{d}} \leq c^{\prime}\|\boldsymbol{v}\|_{H^{1}\left(\Delta_{K}^{\prime}\right)^{d}},
$$

where $\Delta_{K}^{\prime}$ is the union of the $\Delta\left(K^{\prime}\right)$ for $K^{\prime}$ running through $\delta_{K}$. Then,

$$
a_{S}\left(\overline{\boldsymbol{u}}_{h}, \overline{\boldsymbol{v}}_{h}\right) \leq \sum_{K \in \overline{\mathcal{T}}_{h}} \eta_{S K}\|\boldsymbol{v}\|_{H^{1}\left(\Delta_{K}^{\prime}\right)^{d}}
$$


Now standard arguments yield (4.6).

Thanks to the theorem of Pousin and Rappaz [21], we are now in a position to state an upper bound for the error.

Proposition 4.2. If property (2.10) is satisfied, the following a posteriori error estimates hold between a nonsingular solution $(\boldsymbol{u}, p)$ of problem $(2.2)$ and the solution $\left(\overline{\boldsymbol{u}}_{h}, \bar{p}_{h}\right)$ of problem (2.11) associated with it in Theorem 3.6

$$
\left\|\boldsymbol{u}-\overline{\boldsymbol{u}}_{h}\right\|_{H^{1}(\Omega)^{d}}+\left\|p-\bar{p}_{h}\right\|_{L^{2}(\Omega)} \leq c\left(\sum_{K \in \overline{\mathcal{T}}_{h}}\left(\eta_{K}^{2}+\eta_{S K}^{2}+h_{K}^{2}\left\|\boldsymbol{f}-\boldsymbol{f}_{h}\right\|_{L^{2}(K)^{d}}^{2}\right)\right)^{\frac{1}{2}}
$$

Proof: We proceed in two steps.

$1)$ We introduce the following operator $\overline{\mathcal{S}}$ as an extension of $\mathcal{S}$ : With any data $\boldsymbol{f}$ in $H^{-1}(\Omega)^{d}$ and $g$ in $L_{\circ}^{2}(\Omega)$, it associates the part $\boldsymbol{u}$ of the solution $(\boldsymbol{u}, p)$ of the problem

Find $(\boldsymbol{u}, p)$ in $H_{0}^{1}(\Omega)^{d} \times L_{\circ}^{2}(\Omega)$ such that

$$
\begin{array}{lr}
\forall \boldsymbol{v} \in H_{0}^{1}(\Omega)^{d}, & a(\boldsymbol{u}, \boldsymbol{v})+b(\boldsymbol{v}, p)=\langle\boldsymbol{f}, \boldsymbol{v}\rangle, \\
\forall q \in L_{\circ}^{2}(\Omega), & b(\boldsymbol{u}, q)=\int_{\Omega} g(\boldsymbol{x}) q(\boldsymbol{x}) \mathrm{d} \boldsymbol{x} .
\end{array}
$$

Let $\overline{\mathcal{F}}$ be the mapping given by: $\overline{\mathcal{F}}(\boldsymbol{u})=\boldsymbol{u}-\overline{\mathcal{S}}(\mathcal{G}(\boldsymbol{u}), 0)$ (this is just an extension of (3.6)). This function is continuous from $H_{0}^{1}(\Omega)^{d}$ into ifself; moreover the mapping : $\boldsymbol{v} \mapsto D \overline{\mathcal{F}}(\boldsymbol{v})$ is Lipschitz continuous in a bounded neighbourhood of $\boldsymbol{u}$ and $D \overline{\mathcal{F}}(\boldsymbol{u})$ is an isomorphism of $H_{0}^{1}(\Omega)^{d}$. Thus, applying [21, Thm 3] (see also [25, Prop. 2.1]) yields

$$
\left\|\boldsymbol{u}-\overline{\boldsymbol{u}}_{h}\right\|_{H^{1}(\Omega)^{d}} \leq c\left\|\overline{\mathcal{F}}\left(\overline{\boldsymbol{u}}_{h}\right)\right\|_{H^{1}(\Omega)^{d}}=c\left\|\overline{\mathcal{F}}(\boldsymbol{u})-\overline{\mathcal{F}}\left(\overline{\boldsymbol{u}}_{h}\right)\right\|_{H^{1}(\Omega)^{d}} .
$$

By using the continuity of $\overline{\mathcal{S}}$, we obtain from (4.3) and (4.4)

$$
\left\|\boldsymbol{u}-\overline{\boldsymbol{u}}_{h}\right\|_{H^{1}(\Omega)^{d}} \leq c\left(\|\boldsymbol{R}\|_{H^{-1}(\Omega)^{d}}+\left\|R^{*}\right\|_{L^{2}(\Omega)}\right) .
$$

Thanks to Lemma 4.1 and the definition (4.4) of $R^{*}$, we obtain the desired estimate for $\left\|\boldsymbol{u}-\overline{\boldsymbol{u}}_{h}\right\|_{H^{1}(\Omega)^{d}}$.

2) From definition (4.3), we have

$$
b\left(\boldsymbol{v}, p-\bar{p}_{h}\right)=\langle\boldsymbol{R}, \boldsymbol{v}\rangle-a\left(\boldsymbol{u}-\overline{\boldsymbol{u}}_{h}, \boldsymbol{v}\right)-c(\boldsymbol{u}, \boldsymbol{u}, \boldsymbol{v})+c\left(\overline{\boldsymbol{u}}_{h}, \overline{\boldsymbol{u}}_{h}, \boldsymbol{v}\right) .
$$

Thus, owing to the previous estimate and Lemma 4.1, we easily derive the estimate for $\left\|p-\bar{p}_{h}\right\|_{L^{2}(\Omega)}$ from the inf-sup condition (2.3).

However a simpler estimate can be derived with a further non restrictive hypothesis (but without condition (2.10)).

Corollary 4.3. If the space $\mathbb{X}_{h}$ contains the space $\mathbb{X}_{h}^{1}$ defined in (3.2), the following a posteriori error estimate holds between a nonsingular solution $(\boldsymbol{u}, p)$ of problem (2.2) and the solution $\left(\overline{\boldsymbol{u}}_{h}, \bar{p}_{h}\right)$ of the problem (2.11) associated with it in Theorem 3.6

$$
\left\|\boldsymbol{u}-\overline{\boldsymbol{u}}_{h}\right\|_{H^{1}(\Omega)^{d}}+\left\|p-\bar{p}_{h}\right\|_{L^{2}(\Omega)} \leq c\left(\sum_{K \in \overline{\mathcal{T}}_{h}}\left(\eta_{K}^{2}+h_{K}^{2}\left\|\boldsymbol{f}-\boldsymbol{f}_{h}\right\|_{L^{2}(K)^{d}}^{2}\right)\right)^{\frac{1}{2}} .
$$


Proof: We start once again from (4.9). In part 3) of the proof of Lemma 4.1, due to the new assumption, the function $\overline{\boldsymbol{v}}_{h}$ can be taken in $\mathbb{X}_{h}$, so that $\boldsymbol{v}_{h^{\prime}}=\left(\mathrm{Id}-\Pi_{h}\right) \overline{\boldsymbol{v}}_{h}$ is equal to zero and thus the term $a_{S}\left(\overline{\boldsymbol{u}}_{h}, \overline{\boldsymbol{v}}_{h}\right)$ vanishes. All this gives the simplified estimate for $\left\|\boldsymbol{u}-\overline{\boldsymbol{u}}_{h}\right\|_{H^{1}(\Omega)^{d}}$, and the estimate for $\left\|p-\bar{p}_{h}\right\|_{L^{2}(\Omega)}$ follows from exactly the same arguments as previously.

Remark 4.4. The hypothesis that $\overline{\mathbb{X}}_{h}$ contains the space $\mathbb{X}_{h}^{1}$, that yields Lemma 4.1 , is lighter than the hypothesis that $\mathbb{X}_{h}$ contains the space $\mathbb{X}_{h}^{1}$, that yields to Corollary 4.3 . In the last case the error indicators $\eta_{S K}$ are not needed, and we recover the same error indicator as for Navier-Stokes equations.

\subsection{Upper bounds for the indicators.}

In an obvious way, we can write equation (4.3) as

$$
\begin{aligned}
& \sum_{K \in \overline{\mathcal{T}}_{h}}\left(\int_{K}\left(\boldsymbol{f}_{h}+\nu \Delta \overline{\boldsymbol{u}}_{h}-\left(\overline{\boldsymbol{u}}_{h} \cdot \nabla\right) \overline{\boldsymbol{u}}_{h}-\operatorname{grad} \bar{p}_{h}\right) \cdot \boldsymbol{v}(\boldsymbol{x}) d \boldsymbol{x}\right. \\
&\left.+\frac{1}{2} \sum_{e \in \mathcal{E}_{K}} \int_{e}\left[\nu \partial_{h} \overline{\boldsymbol{u}}_{h}-\bar{p}_{h} \boldsymbol{n}\right]_{e}(\boldsymbol{\tau}) \cdot \boldsymbol{v}(\boldsymbol{\tau}) d \boldsymbol{\tau}\right) \\
&=a\left(\boldsymbol{u}-\overline{\boldsymbol{u}}_{h}, \boldsymbol{v}\right)+c(\boldsymbol{u}, \boldsymbol{u}, \boldsymbol{v})-c\left(\overline{\boldsymbol{u}}_{h}, \overline{\boldsymbol{u}}_{h}, \boldsymbol{v}\right)+b\left(\boldsymbol{v}, p-\bar{p}_{h}\right)-\left\langle\boldsymbol{f}-\boldsymbol{f}_{h}, \boldsymbol{v}\right\rangle,
\end{aligned}
$$

for a given unit normal vector $\boldsymbol{n}$ to $e$ and the appropriate sign for $[\cdot]_{e}$. Similarly we have for all $q$ in $L_{\circ}^{2}(\Omega)$

$$
\int_{\Omega}\left(\operatorname{div} \overline{\boldsymbol{u}}_{h}\right)(\boldsymbol{x}) q(\boldsymbol{x}) \mathrm{d} \boldsymbol{x}=b\left(\boldsymbol{u}-\boldsymbol{u}_{h}, q\right) .
$$

Thus bounding the three terms in the indicators $\eta_{K}$ follows from standard arguments that we briefly recall.

Proposition 4.5. Each indicator $\eta_{K}$ defined in (4.1), $K \in \overline{\mathcal{T}}_{h}$, satisfies

$$
\eta_{K} \leq c\left(\left\|\boldsymbol{u}-\overline{\boldsymbol{u}}_{h}\right\|_{H^{1}\left(\omega_{K}\right)^{d}}+\left\|p-\bar{p}_{h}\right\|_{L^{2}\left(\omega_{K}\right)}+\sum_{\kappa \subset \omega_{K}} h_{\kappa}\left\|\boldsymbol{f}-\boldsymbol{f}_{h}\right\|_{L^{2}(\kappa)^{d}}\right),
$$

where $\omega_{K}$ stands for the union of elements of $\overline{\mathcal{T}}_{h}$ that share at least an edge $(d=2)$ or a face $(d=3)$ with $K$.

Proof: We bound successively the three terms in $\eta_{K}$.

1) If $\psi_{K}$ denotes the bubble function on $K$ (equal to the product of the Lagrange coordinates associated with the vertices of $K$ ), we set:

$$
\boldsymbol{v}_{K}= \begin{cases}\left(\boldsymbol{f}_{h}+\nu \Delta \overline{\boldsymbol{u}}_{h}-\left(\overline{\boldsymbol{u}}_{h} \cdot \nabla\right) \overline{\boldsymbol{u}}_{h}-\operatorname{grad} \bar{p}_{h}\right) \psi_{K} & \text { on } K \\ 0 & \text { elsewhere }\end{cases}
$$

Taking $\boldsymbol{v}$ equal to $\boldsymbol{v}_{K}$ (which belongs to $\left.H_{0}^{1}(\Omega)^{d}\right)$ in $(4.11)$ gives

$$
\begin{aligned}
& \left.\|\left(\boldsymbol{f}_{h}+\nu \Delta \overline{\boldsymbol{u}}_{h}-\left(\overline{\boldsymbol{u}}_{h} \cdot \nabla\right) \overline{\boldsymbol{u}}_{h}-\operatorname{grad} \bar{p}_{h}\right)\right) \psi_{K}^{\frac{1}{2}} \|_{L^{2}(K)}^{2} \\
& \quad \leq\left(\left\|\boldsymbol{u}-\overline{\boldsymbol{u}}_{h}\right\|_{H^{1}(K)^{d}}+\left\|p-\bar{p}_{h}\right\|_{L^{2}(K)}\right)\left\|\boldsymbol{v}_{K}\right\|_{H^{1}(K)^{d}}+\left\|\boldsymbol{f}-\boldsymbol{f}_{h}\right\|_{L^{2}(K)^{d}}\|\boldsymbol{v}\|_{L^{2}(K)^{d}} .
\end{aligned}
$$


Using standard inverse inequalities, see [25, Lemma 3.3] for instance, and multiplying by $h_{K}$ thus yield

$$
\begin{aligned}
h_{K} \| \boldsymbol{f}_{h}+\nu \Delta \overline{\boldsymbol{u}}_{h} & -\left(\overline{\boldsymbol{u}}_{h} \cdot \nabla\right) \overline{\boldsymbol{u}}_{h}-\operatorname{grad} \bar{p}_{h} \|_{L^{2}(K)^{d}} \\
& \leq c\left(\left\|\boldsymbol{u}-\overline{\boldsymbol{u}}_{h}\right\|_{H^{1}(K)^{d}}+\left\|p-\bar{p}_{h}\right\|_{L^{2}(K)^{d}}+h_{K}\left\|\boldsymbol{f}-\boldsymbol{f}_{h}\right\|_{L^{2}(K)^{d}}\right) .
\end{aligned}
$$

2) To bound the terms on the edges or faces and, for each $e$ in $\mathcal{E}_{K}$, we introduce a fixed lifting operator $\mathcal{L}_{e, K}$

- that maps functions on $e$ vanishing on $\partial e$ into functions on $K$ vanishing on $\partial K \backslash e$,

- and is constructed from a fixed lifting operator on the reference triangle or tetrahedron. Next, for each edge $e$ in $\mathcal{E}_{K}$ shared by the two elements $K$ and $K^{\prime}$ of $\overline{\mathcal{T}}_{h}$, we set:

$$
\boldsymbol{v}_{e}= \begin{cases}\mathcal{L}_{e, \kappa}\left(\left[\nu \partial_{n} \overline{\boldsymbol{u}}_{h}-\bar{p}_{h} \boldsymbol{n}\right]_{e} \psi_{e}\right) & \text { on } \kappa \in\left\{K, K^{\prime}\right\} \\ 0 & \text { elsewhere }\end{cases}
$$

where $\psi_{e}$ is now the bubble function on $e$. By taking $\boldsymbol{v}$ equal to $\boldsymbol{v}_{e}$ in (4.11) and using the same inverse inequalities as previously and other ones, see once more [25, Lemma 3.3], together with estimate (4.14), we derive

$$
\begin{aligned}
h_{e}^{\frac{1}{2}}\left\|\left[\nu \partial_{n} \overline{\boldsymbol{u}}_{h}-\bar{p}_{h} \boldsymbol{n}\right]_{e}\right\|_{L^{2}(e)^{d}} & \\
& \leq c \sum_{\kappa \in\left\{K, K^{\prime}\right\}}\left(\left\|\boldsymbol{u}-\overline{\boldsymbol{u}}_{h}\right\|_{H^{1}(\kappa)^{d}}+\left\|p-\bar{p}_{h}\right\|_{L^{2}(\kappa)}+h_{\kappa}\left\|\boldsymbol{f}-\boldsymbol{f}_{h}\right\|_{L^{2}(\kappa)}\right) .
\end{aligned}
$$

4) Finally, we set:

$$
q_{K}=\left(\operatorname{div} \overline{\boldsymbol{u}}_{h}\right) \chi_{K},
$$

where $\chi_{K}$ is now the characteristic function of $K$. Taking $q$ equal to $q_{K}$ in (4.12) immediately yields

$$
\left\|\operatorname{div} \overline{\boldsymbol{u}}_{h}\right\|_{L^{2}(K)} \leq\left\|\boldsymbol{u}-\overline{\boldsymbol{u}}_{h}\right\|_{H^{1}(K)^{d}} .
$$

The proposition is now a direct consequence of (4.14), (4.15) and (4.16).

Estimate (4.13) is fully local. But a direct consequence of it is that

$$
\left(\sum_{K \in \overline{\mathcal{T}}_{h}} \eta_{K}^{2}\right)^{\frac{1}{2}} \leq c\left(\left\|\boldsymbol{u}-\overline{\boldsymbol{u}}_{h}\right\|_{H^{1}(\Omega)^{d}}+\left\|p-\bar{p}_{h}\right\|_{L^{2}(\Omega)}+\left(\sum_{K \in \overline{\mathcal{T}}_{h}} h_{K}^{2}\left\|\boldsymbol{f}-\boldsymbol{f}_{h}\right\|_{L^{2}(K)^{d}}^{2}\right)^{\frac{1}{2}}\right) .
$$

When compared with (4.10), this last estimate proves the optimality of the family of indicators $\left(\eta_{K}\right)_{K \in \overline{\mathcal{T}}_{h}}$. Moreover, since estimate (4.13) is local, it can be hoped that these indicators are an efficient tool for adapting the mesh.

Remark 4.6. If the space $\mathbb{X}_{h}$ contains the space $\mathbb{X}_{h}^{1}$ defined in (3.2), the following upper bound can be proved for the indicators $\eta_{S K}, K \in \overline{\mathcal{T}}_{h}$,

$$
\sum_{K \in \overline{\mathcal{T}}_{h}} \eta_{S K}^{\frac{3}{2}} \leq c\left(\frac{h}{h_{\min }}\right)^{1-\frac{d}{4}} \sum_{K \in \overline{\mathcal{T}}_{h}}\left(\eta_{K}+h_{K}\left\|\boldsymbol{f}-\boldsymbol{f}_{h}\right\|_{L^{2}(K)}\right)^{\frac{3}{2}},
$$

where $h_{\text {min }}$ stands for the minimum of the $h_{K}, K \in \mathcal{T}_{h}$. Even if the family of triangulations is uniformy regular (which means that $h_{\min } \geq c h$ ), estimate (4.18) is not fully optimal 
with respect to (4.7) since it involves a bad power of the indicators. Proving local estimates seems rather difficult for general finite elements.

\subsection{A look at the standard subgrid eddy viscosity discretization.}

For a while, we consider the simple mono-scale discrete problem

Find $\left(\overline{\boldsymbol{u}}_{h}, \bar{p}_{h}\right)$ in $\overline{\mathbb{X}}_{h} \times \overline{\mathbb{M}}_{h}$ such that

$$
\begin{aligned}
& \forall \overline{\boldsymbol{v}}_{h} \in \overline{\mathbb{X}}_{h}, \quad a\left(\overline{\boldsymbol{u}}_{h}, \overline{\boldsymbol{v}}_{h}\right)+c\left(\overline{\boldsymbol{u}}_{h}, \overline{\boldsymbol{u}}_{h}, \overline{\boldsymbol{v}}_{h}\right)+b\left(\overline{\boldsymbol{v}}_{h}, \bar{p}_{h}\right) \\
& \quad+\widetilde{a}_{S}\left(\overline{\boldsymbol{u}}_{h}, \overline{\boldsymbol{v}}_{h}\right)=\left\langle\boldsymbol{f}, \overline{\boldsymbol{v}}_{h}\right\rangle, \\
& \forall \bar{q}_{h} \in \overline{\mathbb{M}}_{h}, \quad b\left(\overline{\boldsymbol{u}}_{h}, \bar{q}_{h}\right)=0,
\end{aligned}
$$

where the new Smagorinsky eddy viscosity form $\widetilde{a_{S}}(\cdot, \cdot)$ is now defined by

$$
\widetilde{a_{S}}(\boldsymbol{u}, \boldsymbol{v})=\int_{\Omega} \nu_{S}(\boldsymbol{u})(\boldsymbol{x})(\operatorname{grad} \boldsymbol{u})(\boldsymbol{x}):(\operatorname{grad} \boldsymbol{v})(\boldsymbol{x}) \mathrm{d} \boldsymbol{x} .
$$

By the same arguments as in Section 3, it is readily checked that this problem has a unique solution $\left(\overline{\boldsymbol{u}}_{h}, \bar{p}_{h}\right)$ in a neighbourhood of a nonsingular solution of problem (2.2) which still satisfies the a priori error estimates (3.19) and (3.21).

On the other hand, for each $K$ in $\overline{\mathcal{T}}_{h}$, we introduce the modified error indicator linked to the new Smagorinsky term

$$
\widetilde{\eta_{S K}}=\left(C_{S} h_{K}\right)^{2}\left\|\operatorname{grad} \overline{\boldsymbol{u}}_{h}\right\|_{L^{4}(K)^{d \times d}}^{2}
$$

The same arguments as for Proposition 4.2 lead to the estimate

$$
\left\|\boldsymbol{u}-\overline{\boldsymbol{u}}_{h}\right\|_{H^{1}(\Omega)^{d}}+\left\|p-\bar{p}_{h}\right\|_{L^{2}(\Omega)} \leq c\left(\sum_{K \in \overline{\mathcal{T}}_{h}}\left(\eta_{K}^{2}+{\widetilde{\eta_{S K}}}^{2}+h_{K}^{2}\left\|\boldsymbol{f}-\boldsymbol{f}_{h}\right\|_{L^{2}(K)^{d}}^{2}\right)\right)^{\frac{1}{2}},
$$

and the upper bound (4.13) still holds for the indicators $\eta_{K}$. However, concerning the indicators $\widetilde{\eta_{S K}}$, we can only prove the analogue of (4.18), which is not optimal. This lack of optimality brings to light the interest of using a multi-scale discretization. 


\section{Numerical experiments.}

The computations are performed with the code FreeFem ++ due to Hecht and Pironneau [15]. We have decided here to work with the final spaces ssociated with Taylor-Hood finite elements, see [6, Chap. VI, Example 3.5] or [13, Chap. II, Sect. 4.2] for instance. More precisely, these spaces are defined by

$$
\begin{aligned}
\overline{\mathbb{X}}_{h}=\left\{\boldsymbol{v}_{h} \in H_{0}^{1}(\Omega)^{d} ;\right. & \left.\forall K \in \mathcal{T}_{h},\left.v_{h}\right|_{K} \in \mathcal{P}_{2}(K)^{d}\right\} \\
\overline{\mathbb{M}}_{h} & =\left\{q_{h} \in H^{1}(\Omega) \cap L_{\circ}^{2}(\Omega) ; \forall K \in \mathcal{T}_{h},\left.q_{h}\right|_{K} \in \mathcal{P}_{1}(K)\right\} .
\end{aligned}
$$

Note that the space $\overline{\mathbb{M}}_{h}$ coincides with the space $\mathbb{M}_{h}^{1}$ defined in (3.3). Moreover it is wellknown that the inf-sup condition (3.1) holds for these spaces. We take the space $\mathbb{X}_{h}$ equal to the space $\mathbb{X}_{h}^{1}$ introduced in (3.2), namely

$$
\mathbb{X}_{h}=\left\{\boldsymbol{v}_{h} \in H_{0}^{1}(\Omega)^{d} ; \forall K \in \mathcal{T}_{h},\left.v_{h}\right|_{K} \in \mathcal{P}_{1}(K)^{d}\right\}
$$

and finally the operator $\Pi_{h}$ equal to the Lagrange interpolation operator at all vertices of elements of $\mathcal{T}_{h}$ which are not on the boundary $\partial \Omega$ with values in $\mathbb{X}_{h}$. Nothing more is needed to implement the discrete problem (2.11).

As $\mathbb{X}_{h}$ contains (in fact, is equal to) the space $\mathbb{X}_{h}^{1}$, owing to the error estimate $(4.10)$ we only use the estimators $\eta_{K}$ to perform the grid adaptations.

We have decided to present two numerical experiments. The first one deals with a flow with known smooth solution in order to test the efficiency of the error estimators $\eta_{K}$ and the ability of the grid adaptation process to obtain accurate solutions with reduced computational time. The second one involves a more realistic case (the step flow) to check the ability of the grid adaptation process to accurately solve a flow with low smoothness at large Reynolds number. In both cases we consider laminar flows, the application to fully turbulent flows is in progress.

\subsection{Case of a given solution.}

This test analyzes the efficiency of the error indicators $\eta_{K}$ defined by (4.1). We check whether the error $\left\|\boldsymbol{u}-\boldsymbol{u}_{h}\right\|_{H^{1}(\Omega)}$ is proportional to the Hilbertian sum of the indicators $\left(\sum_{K \in \mathcal{T}_{h}} \eta_{K}^{2}\right)^{1 / 2}$ asymptotically as $h \rightarrow 0$ for small values of the laminar diffusion $\nu$.

We consider the square domain $\Omega=] 0, \pi[\times] 0, \pi[$, and the pair (velocity, pressure) given by

$$
\boldsymbol{u}(x, y)=\left(e^{y} \sin (x),-e^{y} \cos (x)\right), \quad p(x, y)=e^{x+y}+x^{2}+c,
$$

where the constant $c$ is set to have a zero-mean pressure. The pair $(\boldsymbol{u}, p)$ is solution of the Navier-Stokes equations (2.1) with an appropriate r.h.s. $\boldsymbol{f}$ and non-homogeneous Dirichlet boundary conditions. 
The solution of the discrete problem (2.11) has been computed through a single fixed point iteration based upon linearization of this problem. This simple procedure converges without difficulty for all values of $\nu$ tested, $\nu=10^{-1}, \nu=10^{-2}, \nu=10^{-3}$ and $\nu=2 \times 10^{-4}$. The initialization is the solution of the Stokes problem with the same $\nu$. The procedure is assumed to have converged when the relative error between the $L^{2}(\Omega)$-norm of two consecutive iterates is smaller than $10^{-8}$. We have used non-structured meshes to avoid possible super-convergence effects.

We first check the efficiency of the error indicator by computing the efficiency index, i.e., the ratio of the relative error to the Hilbertian sum of the indicators. Tables 1 to 4 display these results corresponding to the four values of the Reynolds numbers, respectively, for increasing numbers of degrees of freedom.

\begin{tabular}{|c|c|c|c|c|}
\hline Degrees of freedom & CPU Time & Relative Error & Sum Indicators & Efficiency \\
\hline 1192 & 18.44 & 0.076481 & 19.4276 & 0.003937 \\
4460 & 68.05 & 0.031235 & 4.04622 & 0.007720 \\
9897 & 125.05 & 0.016780 & 1.931050 & 0.008690 \\
17728 & 195.66 & 0.010158 & 1.030970 & 0.009853 \\
27305 & 325.99 & 0.006380 & 0.641225 & 0.009950 \\
39159 & 440.47 & 0.005159 & 0.479480 & 0.010761 \\
52534 & 573.74 & 0.004103 & 0.368128 & 0.011147 \\
68708 & 685.35 & 0.003551 & 0.293501 & 0.012100 \\
85665 & 768.25 & 0.002753 & 0.241493 & 0.011398 \\
108184 & 967.91 & 0.002078 & 0.183102 & 0.011347 \\
\hline
\end{tabular}

Table 1. Efficiency for $\nu=10^{-1}$

\begin{tabular}{|c|c|c|c|c|}
\hline Degrees of freedom & CPU Time & Relative Error & Sum Indicators & Efficiency \\
\hline 1192 & 25.62 & 0.137147 & 23.2329 & 0.005903 \\
4460 & 105.19 & 0.076050 & 5.269140 & 0.014433 \\
9897 & 185.15 & 0.047807 & 2.853680 & 0.016753 \\
17728 & 350.90 & 0.035531 & 1.781520 & 0.019944 \\
27305 & 517.89 & 0.025477 & 1.169900 & 0.0217777 \\
39159 & 696.21 & 0.020265 & 0.893653 & 0.022676 \\
52534 & 1086.01 & 0.036521 & 1.454960 & 0.025101 \\
68708 & 1123.8 & 0.014649 & 0.620200 & 0.023620 \\
85665 & 1500.64 & 0.011526 & 0.491341 & 0.0234587 \\
108184 & 1790.11 & 0.009919 & 0.423223 & 0.023437 \\
\hline
\end{tabular}

Table 2. Efficiency for $\nu=10^{-2}$ 


\begin{tabular}{|c|c|c|c|c|}
\hline Degrees of freedom & CPU Time & Relative Error & Sum Indicators & Efficiency \\
\hline 1192 & 26.65 & 0.151984 & 23.9895 & 0.006335 \\
4460 & 113.71 & 0.100590 & 5.990380 & 0.016792 \\
9897 & 210.73 & 0.070909 & 3.692480 & 0.019204 \\
17728 & 396.32 & 0.060863 & 2.759200 & 0.022058 \\
27305 & 588.75 & 0.052434 & 2.190070 & 0.023942 \\
39159 & 853.88 & 0.044483 & 1.791170 & 0.024835 \\
52534 & 1086.01 & 0.036521 & 1.454960 & 0.025101 \\
68708 & 1389.95 & 0.034326 & 1.351890 & 0.025391 \\
85665 & 1864.85 & 0.028890 & 1.184070 & 0.024399 \\
108184 & 2168.61 & 0.026960 & 1.063480 & 0.025350 \\
\hline
\end{tabular}

Table 3. Efficiency for $\nu=10^{-3}$

\begin{tabular}{|c|c|c|c|c|}
\hline Degrees of freedom & CPU Time & Relative Error & Sum Indicators & Efficiency \\
\hline 1192 & 27.23 & 0.153598 & 24.0634 & 0.006383 \\
4460 & 121.41 & 0.104726 & 6.089710 & 0.017197 \\
9897 & 225.29 & 0.076238 & 3.858290 & 0.019759 \\
17728 & 430.01 & 0.066984 & 2.968460 & 0.022565 \\
27305 & 684.72 & 0.061366 & 2.504370 & 0.024504 \\
39159 & 965.48 & 0.053840 & 2.197200 & 0.024504 \\
52534 & 1366.51 & 0.046629 & 1.805160 & 0.025831 \\
68708 & 1611.31 & 0.044679 & 1.716120 & 0.026035 \\
85665 & 2248.91 & 0.040041 & 1.520500 & 0.026334 \\
108184 & 2962.29 & 0.039491 & 1.580815 & 0.024981 \\
\hline
\end{tabular}

Table 4. Efficiency for $\nu=2 \times 10^{-4}$

In all cases the efficiency index tends to a constant as the number of degrees of freedom increases. This is made apparent in Figure 1, that displays the efficiency index for all cases considered. 


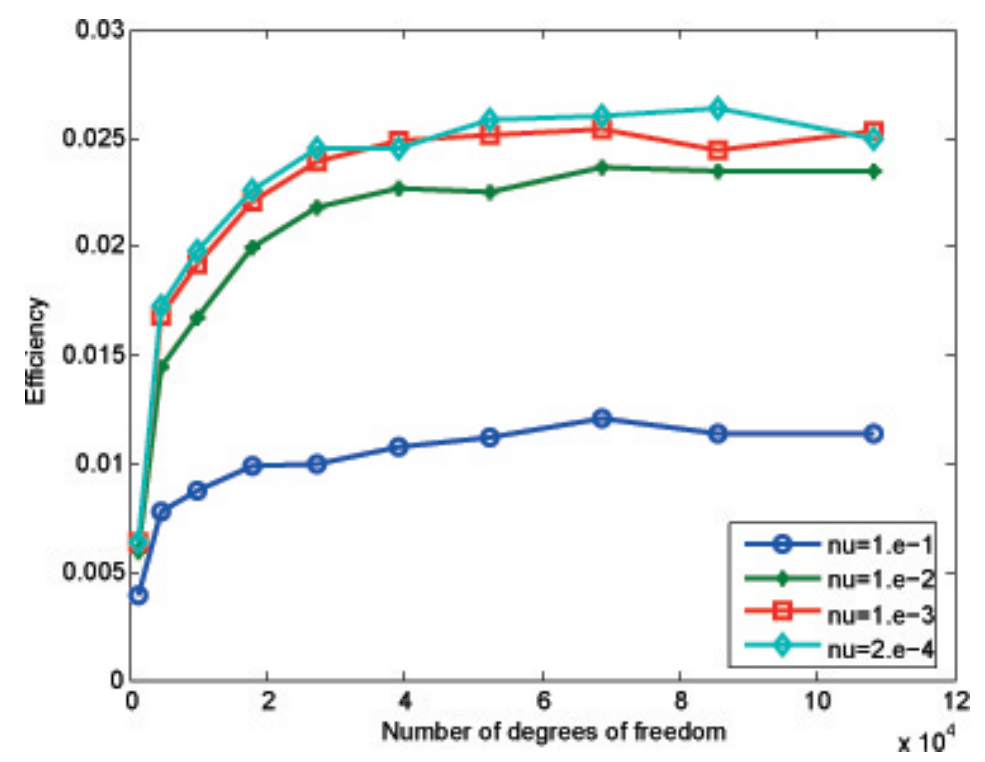

Figure 1. Efficiency of the indicators

\begin{tabular}{|c|c|c|c|c|c|c|}
\hline$R e$ & $\nu$ & $\begin{array}{c}\text { Relative Error } \\
\text { (direct) }\end{array}$ & $\begin{array}{c}\text { CPU Time } \\
\text { (direct) }\end{array}$ & $\begin{array}{c}\text { Relative Error } \\
\text { (with adaptation) }\end{array}$ & $\begin{array}{c}\text { CPU Time } \\
\text { (with adaptation) }\end{array}$ & Gain \\
\hline 10 & $10^{-1}$ & 0.002078 & 967.91 & 0.001785 & 248.68 & 3.9 \\
100 & $10^{-2}$ & 0.020265 & 696.21 & 0.020538 & 311.16 & 2.2 \\
100 & $10^{-2}$ & 0.009919 & 1790.11 & 0.008803 & 469.35 & 3.8 \\
1000 & $10^{-3}$ & 0.036521 & 1086.01 & 0.037762 & 370.63 & 2.9 \\
1000 & $10^{-3}$ & 0.026960 & 2168.61 & 0.017130 & 1104 & 2.0 \\
5000 & $210^{-4}$ & 0.046629 & 1366.51 & 0.04766 & 431.62 & 3.2 \\
5000 & $210^{-4}$ & 0.039491 & 2962.29 & 0.028350 & 1548.02 & 1.9 \\
\hline
\end{tabular}

Table 5. Comparison of CPU times, direct versus adaptive solution

We also have compared the CPU time required to achieve an error below a certain tolerance for all considered Reynolds numbers, between the direct calculation in a fixed grid and the adaptive calculation. The results are displayed in Table 5. The CPU time gain ranges from 2 to 4, essentially depending on the number of grid adaptations required to achieve the prescribed tolerance.

\subsection{A more realistic case.}

In this test we analyze the performance of our adaptive strategy to solve a well known, but rather hard to solve, problem. This is the backward-facing step flow. This flows takes place in a non-convex domain, and consequently the velocity and pressure have a low accuracy. In this flow the challenge is to accurately compute the reattachment length, here denoted by $x_{1}$, which is the length of the main recirculating region behind the step. We refer to [1] and [10] for a more detailed description of the difficulties linked to this problem. 
The characteristics of the backward-facing step flow considered in this study are shown in Figure 2, where $h_{l}=5.2, H=10.1, h=4.9$.

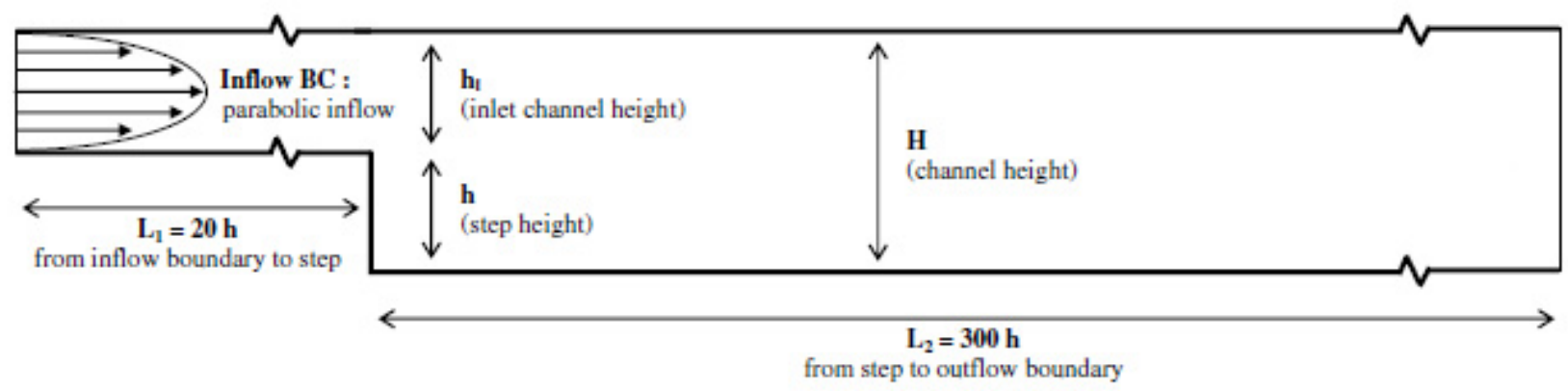

Figure 2. Geometric characteristics of backward-facing step problem

The Reynolds number is defined as $R e=\frac{U D}{\nu}$, where $U$ is the inlet mean velocity or in other words two third of the maximum inlet velocity and $D$ is the hydraulic diameter of the inlet channel $D=2 h_{l}$. To change the Reynolds number, we set the value of the laminar viscosity to $\nu=15$, and we re-scale the inlet velocity profile.

\begin{tabular}{|c|c|c|c|}
\hline$R e$ & $\begin{array}{c}\text { Our } x_{1} \\
\text { (without adapt.) }\end{array}$ & $\begin{array}{c}\text { Our } x_{1} \\
\text { (with adapt.) }\end{array}$ & $\begin{array}{c}\text { Ref. }[1] \\
\text { (experimental) }\end{array}$ \\
\hline 100 & 3.01114 & 3.00439 & 2.98 \\
300 & 6.38367 & 6.66069 & 6.66 \\
500 & 9.68886 & 9.91935 & 10.10 \\
1000 & 17.55904 & 17.70518 & 17.86 \\
\hline
\end{tabular}

Table 6. Comparison of reattachment points

The single fixed point iteration used in Section 5.1 fails here, even for moderate Reynolds numbers. Instead, we have used a Newton method with a continuation strategy with respect to the Reynolds number: The initialization to compute $R e=100$ is the solution for $R e=10$, and so on for all values considered: $R e=100, R e=300, R e=500$ and $R e=1000$. For larger values the flow becomes unsteady, up to $R e \simeq 8.000$, where the flow becomes fully turbulent.

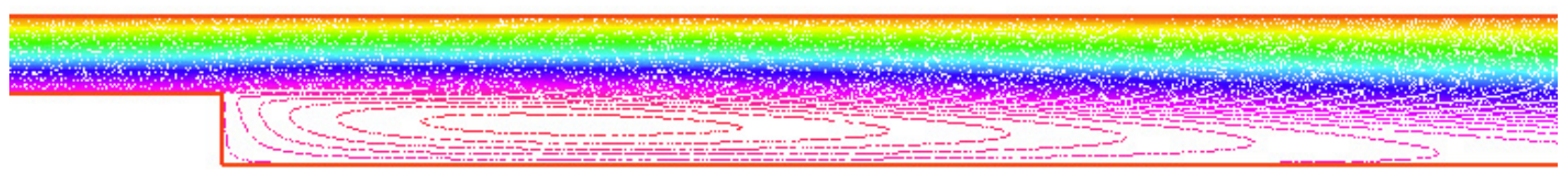

Figure 3. Streamlines of the adaptive solution for $R e=1000$

The computation for non-adapted grids also uses a continuation strategy with respect to the Reynolds number, where the initialization for the current Reynolds number is the solution obtained with the adapted grid with the preceding Reynolds number. If instead of this initialization we use the solution with the non-adapted grid for the preceding Reynolds 
number, the Newton's method does not converge. Then, the comparison of CPU times is not meaningful. Let us say, anyhow, that these times are similar, but the precision obtained with the adaptive procedure is better than the one obtained with the direct solution (see Table 6). 


\section{References}

[1] B.F. Armaly, F. Durst, J.C.F. Pereira, D. Schöung — Experimental and theoretical investigation of backward-facing step flow, J. Fluid. Mech 127 (1983), 473-496.

[2] C. Bernardi, T. Chacón Rebollo, F. Hecht, R. Lewandowski - Automatic insertion of a turbulence model in the finite element discretization of the Navier-Stokes equations, Math. Models and Methods in Applied Sciences 19 (2009), 1139-1183.

[3] C. Bernardi, F. Coquel, P.-A. Raviart - Automatic coupling and finite element discretization of the Navier-Stokes and heat equations, submitted.

[4] C. Bernardi, Y. Maday, F. Rapetti - Discrétisations variationnelles de problèmes aux limites elliptiques, Collection "Mathématiques et Applications" 45, Springer-Verlag (2004).

[5] L.C. Berselli, T. Iliescu, W.J. Layton - Mathematics of Large Eddy Simulation of Turbulent Flows, Springer (2006).

[6] F. Brezzi, M. Fortin - Mixed and Hybrid Finite Element Methods, Springer-Verlag (1991).

[7] F. Brezzi, J. Rappaz, P.-A. Raviart - Finite dimensional approximation of nonlinear problems, Part I: Branches of nonsingular solutions, Numer. Math. 36 (1980), 1-25.

[8] T. Chacón Rebollo, R. Lewandowski - Mathematical and Numerical Foundations of Turbulence Models and Applications, in preparation.

[9] M. Dauge - Stationary Stokes and Navier-Stokes systems on two- or three-dimensional domains with corners I, SIAM Journal on Mathematical Analysis 20 (1989), 74-97.

[10] E. Erturk - Numerical solutions of 2-D steady incompressible flow over a backward-facing step, Part I: High Reynolds number solutions, Computer \& Fluids 37 (2008), 633-655.

[11] M. Germano - Differential filters for the large eddy numerical simulation of turbulent flows, Phys. Fluids 29(6) (1986), 1755-1757.

[12] M. Germano - Differential filters of elliptic type, Phys. Fluids 29(6) (1986), 1757-1758.

[13] V. Girault, P.-A. Raviart - Finite Element Methods for Navier-Stokes Equations, Theory and Algorithms, Springer-Verlag (1986).

[14] P. Grisvard - Elliptic Problems in Nonsmooth Domains, Pitman (1985).

[15] F. Hecht, O. Pironneau - FreeFem++, second edition, v 3.15, Université Pierre et Marie Curie, Paris (2011), on the web at http://www.freefem.org/ff++/ftp/freefem++doc.pdf.

[16] T. J. R. Hughes, G. Feijóo, L. Mazzei, J-B Quincy - The variational multiscale method: a paradigm for computational mechanics. Comp. Meth. Appl. Mech. Enrgrg. 166 (1-2) (1998), $3-24$.

[17] T. J. R. Hughes, L. Mazzei, K. E. Jansen - Large eddy simulation and the variational multiscale method, Comput. Vis. Sci. 3 (2000), 47-59.

[18] T. J. R. Hughes, L. Mazzei, A. Oberai, A. Wray - The multiscale formulation of large eddy simulation, Phys. Fluids 13(2) (2001), 505-512.

[19] T. J. R. Hughes, A. Oberai, L. Mazzei - Large eddy simulation of turbulent channel flows by the variational multiscale method, Fluids 13(6) (2001), 1784-1799.

[20] D. K. Lilly - A proposed modification of the Germano subgrid closure method, Physics of Fluids A 4(3) (1992), 633-635. 
[21] J. Pousin, J. Rappaz - Consistency, stability, a priori and a posteriori errors for PetrovGalerkin methods applied to nonlinear problems, Numer. Math. 69 (1994), 213-231.

[22] J. Smagorinsky - General circulation experiment with the primitive equations. I. The basic experiment, Monthly Weather Review 91 (1963), 99-164.

[23] R. Temam - Navier-Stokes Equations, AMS Chelsea (2001).

[24] E. R. VanDriest - On turbulent flow near a wall, J. Aerosp. Sci. 23 (1956),1007.

[25] R. Verfürth - A Review of A Posteriori Error Estimation and Adaptive Mesh-Refinement Techniques, Wiley \& Teubner (1996). 